\title{
WestVirginiaUniversity
}

THE RESEARCH REPOSITORY @ WVU

Graduate Theses, Dissertations, and Problem Reports

2010

\section{Transfer and Persistence of Gunshot Residue Particles}

DeAnna M. Wallace

West Virginia University

Follow this and additional works at: https://researchrepository.wvu.edu/etd

\section{Recommended Citation}

Wallace, DeAnna M., "Transfer and Persistence of Gunshot Residue Particles" (2010). Graduate Theses, Dissertations, and Problem Reports. 4667.

https://researchrepository.wvu.edu/etd/4667

This Thesis is protected by copyright and/or related rights. It has been brought to you by the The Research Repository @ WVU with permission from the rights-holder(s). You are free to use this Thesis in any way that is permitted by the copyright and related rights legislation that applies to your use. For other uses you must obtain permission from the rights-holder(s) directly, unless additional rights are indicated by a Creative Commons license in the record and/ or on the work itself. This Thesis has been accepted for inclusion in WVU Graduate Theses, Dissertations, and Problem Reports collection by an authorized administrator of The Research Repository @ WVU. For more information, please contact researchrepository@mail.wvu.edu. 


\title{
Transfer and Persistence of Gunshot Residue Particles
}

\author{
by
}

DeAnna M. Wallace, B.S

Thesis submitted to the

Eberly College of Arts and Sciences

at West Virginia University

in partial fulfillment of the requirements

for the degree of

Master of Science

in

Forensic and Investigative Science

Approved by

Dr. Keith Morris, Ph.D, Chairperson

Mrs. Tina Moroose, M.S.F.S

Dr. Suzanne Bell, Ph.D

Forensic and Investigative Science Program

Morgantown, West Virginia

2010

Keywords: gunshot residue, transfer, persistence, secondary transfer, SEM/EDS, GSR

DeAnna M. Wallace, B.S. 


\title{
Abstract \\ Transfer and Persistence of Gunshot Residue Particles
}

\author{
by DeAnna M. Wallace, B.S
}

In the U.S., although this technique has been in use for many years, there is still debate as to what constitutes GSR. When the firing pin strikes the primer, the priming compound is compressed and detonates since it is sensitive to percussion. This, in turn, causes the propellant to ignite and build up pressure within the cartridge case. When sufficient pressure is built up the bullet will be forced down and out of the barrel. The compounds chosen for this formulation are mainly inorganic in nature. Many of the components will be vaporized during ignition because of the extremely high temperatures. Upon the exit of the bullet from the barrel, the gases and vapors will escape through the various openings. When these vapors are exposed to a rapid decrease in pressure and temperature, they will condense and the generated particles will be deposited on the person of the shooter or someone in close proximity. Particles that land on the shooter are collected and analyzed using the scanning electron microscope/energy dispersive spectroscopy (SEM/EDS). The objective of the study was to evaluate the persistence of gunshot residue particles on the hands of a shooter. Shots were fired by four volunteers who were sampled for GSR at various times after the shooting. A $1 \mathrm{~cm}$ piece of carbon tape (Ted Pella Inc) was placed on a $1 \mathrm{~cm}$ aluminum stub and samples were collected from the back of the hand. These samples were analyzed using the SEM/EDS. The persistence of unique gunshot residue particles over a time frame of 0180 minutes was evaluated. Analyses of these 22 samples from two of the volunteers using the $9 \mathrm{~mm}$ pistol were analyzed by manual analysis. Results show that over the selected time frames there is a decrease in the number of unique GSR particles collected on the hands of a shooter after approximately one hour. 


\section{Dedication}

The author wishes to dedicate this research to the victims who may benefit from gunshot residue examination, including persons who may have been wrongfully convicted. 


\section{Acknowledgments}

I take this moment to acknowledge all of those who made this achievement possible.

Thank you West Virginia University's Forensic \& Investigative Science Program for having faith in me these past six years. As I obtained a Bachelors of Science in Forensic \& Investigative Science during my undergraduate, while continuing to become part of the first graduating class to obtain a Master of Science in Forensic and Investigative Science as an Examiner.

Thank you Dr. Keith Morris, Ph.D, my advisor throughout this study. Without your help and dedication I never would have finished this project. Thank you for you countless hours of time to help me achieve my goal and be a member of the first Masters' graduating class.

Thank you committee members: Mrs.Tina Moroose and Dr. Suzanne Bell. You constantly pushed me to the next level as a person, as a writer, and as a scientist.

In addition, a thank you goes to the volunteers who graciously donated their time to shoot so that I could collect samples. For without your time, this study never would have been possible.

To my family, six years ago you dropped me off at Boreman Hall without knowing a soul. Bringing me back and forth to and from campus when I was homesick we made it all possible. I believed in myself only because you believed in me. To my friends, thank you all for providing me a sense of relief when I needed it most. To Jason, thank you for always being there loving and supporting me and most importantly providing me sanity through challenging times. I love you all.

Thanks again everyone! 


\section{List of Figures}

Figure 1: A positive GSR particle on sample 3 of study, spherical morphology..........5

Figure 2: Diagram of electron energy levels and their respective series................12

Figure 3: Example table of concentration of unique gunshot residue particles vs. time...18

Figure 4: Representation of the firing cycle of a firearm...........................24

Figure 5: Area of the hands sampled by the carbon SEM stubs....................41

Figure 6: Collection method for the persistence of gunshot residue study $\ldots \ldots \ldots \ldots \ldots . . .42$

Figure 7: Automated scanning setup parameters.................................44

Figure 8: Quant Optimization step of the automated scan setup......................45

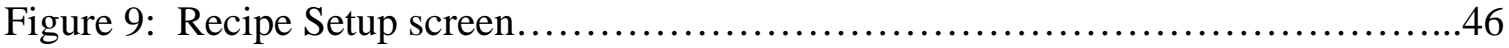

Figure 10: Area Layout screen with all seven stubs selected for analysis...............48

Figure 11: Feature detection screen to set threshold and calibration...................49

Figure 12: Detection setup used to set field and features.........................50

Figure 13: Spectrum setup used to time frames for spectrum analysis.................51

Figure 14: Run stage.....................................................52

Figure 15: Review classes................................................53

Figure 16: Number of unique GSR particles vs. time for participant $1 \ldots \ldots \ldots \ldots \ldots \ldots \ldots . .58$

Figure 17: Number of unique GSR particles vs. time for participant 3.................59

Figure 18: Number of unique GSR particles vs. time for participant 3............... 60 not including time zero datum point

Figure 19: Number of indicative GSR particles vs. time for participant 1 ..............62

Figure 20: Number of indicative GSR particles vs. time for participant 3.............63

Figure 21: Scatter Plot of particle classifications looking at the relationship............65 between area and shape of the particles 
Figure 22: Scatter Plot of shape of the particle for each element in each...............67 particle classifications

Figure 23: Scatter Plot of grey scale of the particle for each element in.................69 each particle classifications 


\section{List of Tables}

Table 1: Particle classification scheme for classifying gunshot residue..................5 defined by the INCA 2008 Software

Table 2: Classification scheme used in the Gialamas study..........................35

Table 3: Firearm information...................................................43

Table 4: Ammunition used in this study.....................................43

Table 5: Operating conditions for the SEM/EDS $\ldots \ldots \ldots \ldots \ldots \ldots \ldots \ldots \ldots \ldots \ldots \ldots \ldots . . .44$

Table 6: Identification numbers for stub to sample analysis for participant $1 \ldots \ldots \ldots \ldots . . .54$

Table 7: Identification numbers for stub to sample analysis for participant 3............54

Table 8: Participant 1 number of particles for each classification at each interval........55

Table 9: Participant 3 number of particles for each classification at each interval........55

Table 10: Total number of particles for each classification at each interval.............56

Table 11: Calculated data from Excel for Participant 1 using first order kinetics.........57 for unique GSR particles

Table 12: Calculated data from Excel for Participant 3 using first order kinetics.........59 for unique GSR particles

Table 13: Calculated data from Excel for Participant 1 using first order kinetics.........62 for indicative GSR particles

Table 14: Calculated data from Excel for Participant 3 using first order kinetics.........63 for indicative GSR particles 


\section{Chapter 1: Introduction}

\subsection{Purpose}

Gunshot residue (GSR) is one of the most underutilized types of physical evidence. The Federal Bureau of Investigation cites firearms being a weapon of choice in over 510,000 violent crimes (1). With this choice of weapon comes a vital piece of evidence. Gunshot residue particles are formed during the discharge of a firearm. Although this technique has been in use for many years, there is till debate as to what constitutes GSR. How many particles, consisting of lead, barium, and antimony, are needed to be considered a positive test for gunshot residue? What if these particles do not contain one of the three key elements? Is the presence of a particle on an individual due to primary or secondary transfer? Normally the presence of a GSR particle on an individual would indicate that this individual has discharged a firearm, or that they have been in close proximity to the discharge of a firearm. Is this really the case?

When the firing pin strikes the primer, the priming compound is compressed and detonates since it is sensitive to percussion. This, in turn, causes the propellant to ignite and build up pressure within the cartridge case. When sufficient pressure is built up the bullet will be forced down and out of the barrel. The primer is formulated to ensure its rapid ignition. The compounds chosen for this formulation are mainly inorganic in nature. Many of the components will be vaporized during ignition because of the extremely high temperatures. Upon the exit of the bullet from the barrel, the gases and vapors will escape through the various openings. When these vapors are exposed to a rapid decrease in pressure and temperature, they will condense and the generated particles will be deposited on the person of the shooter or someone in close proximity (2). 
As the bullet passes through the barrel, portions of the bullet and/or jacket are removed and can be melted and vaporized. Particles which are deposited on the shooter cam be collected and analyzed using a scanning electron microscope with an energy dispersive spectroscopy capability (SEM/EDS). The SEM/EDS method allows for the detection and analysis of each individual particle in the sample. Most other methods for the analysis of gunshot residue are bulk analysis rather than particle analysis. Particle analysis is the recommended method because one can determine both the composition and morphology of individual particles. Bulk analysis on the other hand only provides the relative concentrations of each of the analytes irrespective of their source. This reinforces the value of the SEM/EDS method since the size, morphology, and composition of each individual particle can be determined. These data will allow the analyst to interpret the results.

GSR particles expelled from a firearm tend to have a specific morphology and size range. It has been reported that between $70-100 \%$ of particles in a sample of gunshot residue are spheroidal (3). They may also be stretched, dented, or otherwise distorted, but three-dimensional roundedness is a characteristic of their classification. The surface of the spheroid may be smooth or fuzzy, scaly, or even covered with smaller spheroids. Occasionally, they are capped, perforated, broken, or stemmed. The vast majority of the detected spheroidal particles have diameter of less then $5 \mu \mathrm{m}$ (3).

If a sample contains particles which meet the requirements of being gunshot residue, can an analyst determine the time of firing or whether the individual being tested was the shooter or simply in close proximity to the gun when the shot was fired? The answers to these questions are critical to the interpretation of GSR as an evidence type. 
The objective of the study is to evaluate the persistence of GSR from a primary disposition to the hands of the shooters. Using this information one can determine if there is a relationship between the loss of particles and time (persistence).

During the Chicago Study (4), samples were collected from 201 police vehicles and various detention facilities using GSR kits. Of these samples, 178 lacked the three component primer residue particles. The remaining 23 samples had a total of 56 threecomponent primer residue particles (there was no indication of how these particles were distributed amongst the 23 samples). The authors concluded that there was a possibility of secondary transfer of GSR particles to the hands of suspects who have been transported or detained in an environment contaminated with GSR particles (5). There was no actual testing of human subjects who were exposed to such environments to prove this hypothesis. Other studies which have been performed on the persistence and transfer of gunshot residue include:

- Time period of GSR particles deposition after discharge (3)

- Hair combing to collect organic gunshot residues (6)

- Distribution of GSR particles in the surroundings of a shooting pistol (5)

According to Fojtàšek et al. the maximum number of GSR particles found during a study of their spatial distribution was in the right front quadrant at a distance of 2-4m with respect to the firearm position and shooting direction (5). GSR particles were even found at a distance of $10 \mathrm{~m}$ from firearm (5). The distribution of GSR particles in seven directions was studied both indoors and outdoors using two different primer types. 
During a study of the settling time of GSR particles, it was determined that after the discharge of a pistol, all particles had settled within 8 minutes (7).

The aim of the present study differs from the above studies in that it attempted to quantify the persistence of GSR. The previous studies simply evaluated the presence of GSR particles in environments where suspects in cases would be located and settling times of particles.

\subsection{Background}

It is well known that the fire triangle consists of a fuel, oxygen, and heat. This concept may be extrapolated to a primer mixture. A small arms primer generally consists of an explosive, oxidizer, fuel, and a frictionator. Other compounds may be added to act as sensitizers and binders. The function of a sensitizer is to sensitive the mixture to the percussive force of the firing pin on the primer. The oxidizer must supply at least enough oxygen for the complete combustion of the primer. The components of a primer should be chosen in such a way as to limit the negative effects of the combustion products. There may be more than one explosive, oxidizer, fuel, and frictionator in a single priming composition (8).

A typical modern priming mixture will contain lead styphnate $\left(\mathrm{PbC}_{6} \mathrm{HN}_{3} \mathrm{O}_{8}\right)$ as the explosive, barium nitrate $\left(\mathrm{Ba}\left(\mathrm{NO}_{3}\right)_{2}\right)$ as the oxidizer, antimony sulfide $\left(\mathrm{Sb}_{2} \mathrm{~S}_{3}\right)$ as the fuel, and tetracene $\left(\mathrm{C}_{18} \mathrm{H}_{12}\right)$ as the sensitizer. From this mixture the resulting inorganic products of the reaction will be lead, barium, antimony, and small amounts of sulfur (8). 


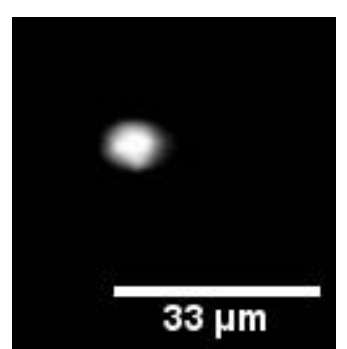

Figure 1: A positive GSR particle on sample 3 of study, spherical morphology

During the discharge of a firearm and the resultant condensation of the vapors, a number of different mixtures may be formed (8). In this study the following classification scheme was employed.

\begin{tabular}{|c|c|c|}
\hline Unique & Indicative & Environmental \\
\hline $\mathrm{SnSbBaPb}$ & $\mathrm{SbBa}$ & $\mathrm{Sb}$ \\
$\mathrm{SbBaPb}$ & $\mathrm{SbPb}$ & $\mathrm{Cu} \mathrm{Zn}$ \\
$\mathrm{SbSnPb}$ & $\mathrm{SbSn}$ & $\mathrm{Ni}$ \\
$\mathrm{SbSnBa}$ & $\mathrm{BaPb}$ & $\mathrm{Pb}$ \\
$\mathrm{Ti} \mathrm{Zn} \mathrm{Cu}$ & $\mathrm{Sr}$ & $\mathrm{Sn}$ \\
$\mathrm{SnBaPb}$ & $\mathrm{Ti} \mathrm{Zn}$ & $\mathrm{Au}$ \\
$\mathrm{Ti} \mathrm{Zn} \mathrm{Sn}$ & & lighter flint \\
& & $\mathrm{Fe}$ \\
& & $\mathrm{Cu}$ \\
\hline
\end{tabular}

Table 1: Particle classification scheme for classifying gunshot residue defined by the INCA 2008 Software.

All particles analyzed during this study were classified according to the scheme, while any particle which did not fulfill the requirements for one of these sets was classified as unclassified. Particles classified as unique were considered to be positive gunshot residues. Indicative particles are gunshot residue particles which contain two of the three main elements. Indicative particles indicate the discharge of a firearm but can not do so unequivocally. An environmental classification is that set of particles which 
are known to the present in the environment. These particles could also be products of a discharge but have no real putative value.

It is important to consider the number and distribution of all classes of particles found in any sample. The composition, shape, size, and appearance as well as the range of particle types should all be considered during interpretation of GSR.

The SEM/EDS not only has the ability to image, but also to analyze materials that ordinarily would not be observed when using an optical microscope. The SEM can produce images of high resolution. This means that closely spaced features on a sample can be resolved into its component parts in an SEM. Imaging refers to the characteristic three-dimensional appearance useful for understanding the surface structure and morphological information of a sample.

Once a sample has been collected, it is prepared and mounted on a stub for examination by SEM/EDS. The SEM uses a focused scanning electron beam to produce images of the sample. These images of the sample are formed point by point through the use of scanning coils which cause the electron beam to move along a set series of points to form a raster. At each point the electron beam interacts with the sample and generates a signal. The scan generator controls this movement and using the acquired signal generates an image on the screen. The size of the image on the screen relative to the scanned area on the sample is used to determine the magnification of the image. The primary electron beam interacts with the sample in a number of ways, the key interactions being: 
- Generation of low energy secondary electrons which allow for the imaging of the topography of the specimen.

- Generation of backscattered electrons which allow for images with contrast based on the average atomic number (Z) of the sample (8).

Electrons impacting on the sample will interact with the sample. These interactions can take place either on the surface or deeper into the bulk of the sample. The intensity of the resulting signals will be a function of the depth at which they originated. It has been determined that an electron beam with a $1 \mu \mathrm{m}$ diameter interacting with a low density, low atomic number sample will produce an interaction volume at 20 $\mathrm{keV}$ with an overall dimension of several micrometers (9). The energy deposition rate varies rapidly throughout the interaction volume, decreasing outwards from the impact point. When mapping the lowest energy deposition contours, a pear shaped volume is obtained.

Some electron specimen interactions are elastic while others are inelastic. An inelastic interaction results in a loss of energy. Electrons which are loosely bound outershell electrons in the atoms of the sample can be ejected from the atom when the energy they receive during this inelastic scatter is sufficient. These electrons are known as secondary electrons. When they are ejected and set into motion, they propagate through the sample and can escape through the surface. A secondary electron is defined as any electron which escapes from the surface of the sample and has a kinetic energy less then $50 \mathrm{eV}$. The detection of these electrons is the standard detection mode in SEM/EDS analysis (secondary electron imaging or SEI). The image thus created is a readily 
interpretable image of the surface. SEI can produce very high-resolution images and contrast is determined by the sample morphology. Details in the 1 to $5 \mathrm{~nm}$ size range can be successfully imaged.

Backscattered electrons (BSE) are beam electrons which have intercepted the sample surface and subsequently escape the specimen. In general, these electrons undergo many elastic scatterings to change their trajectory such that they are able to return to the surface. Monte Carlo trajectory plots for different elements under varying operating conditions indicate that backscattering increases with increased atomic number (9). The inherent advantage of this property allows for the calibration of grayscale as a function of atomic number. The extent of backscatter is also dependent upon the energy of the electron beam. The nature of this dependency is quite complex. The resolution in a BSE image is lower than that of a SEI image given that the interaction volume is pear shaped and BSEs are generated deeper in the sample.

According to Schwoeble, small particles of quartz and feldspar derived from soil will be relatively dark compared to $\mathrm{Pb}, \mathrm{Ba}$, and $\mathrm{Sb}$ particles derived from GSR. When looking at a sample of GSR, examination would be in the BSE mode thus passing up the darker particles while singling out the brighter GSR particles (8) .

During a normal electron beam sample interaction both BSEs and SEs can be generated. Both of these types of electrons can be detected simultaneously. In a classic Everhart-Thornley detector, a positive bias (+ 100V) is placed on a collector grid which is in front of a scintillation counter ( $+12 \mathrm{kV}$ bias) (9). This signal is ultimately detected and amplified to form an SE image. It must be noted that under these conditions BSEs 
can also be detected. To form a BSE image the collector grid receives a negative bias (300V). Under these conditions the low energy SE are repelled by the collector whilst the high energy electrons interact with the scintillation device because of its high bias.

The imaging mode of the SEM/EDS is dependent upon factors such as: electron probe size, electron probe current, electron probe convergence angle, and electron beam accelerating voltage.

In imaging there are four modes of operation:

- Resolution mode requires sufficient beam current to result in image. This mode calls for a small probe size and has high resolution, but is only meaningful at high magnifications.

- High current mode is best for imaging visibility and quality, but a large beam of current is required.

- Depth of focus mode requires small electron probe convergence angles. With a small convergence angle the beam diameter undergoes only a small change over a variety of vertical distances.

- Low voltage mode typically has accelerating voltage less than $5 \mathrm{kV}$. Under these conditions the beam-specimen interactions occur very close to the surface of the specimen. With a high accelerating voltage (15-30 $\mathrm{kV}$ ), the beam penetrates the surface and the signals are from the interior of the sample where inner electrons are excited. 
Compared to optical microscopy, the depth of field is considerably greater in the SEM with the entire particle remaining in focus due to a small aperture. Modern SEM/EDS systems provide for the capture and manipulation of the images and spectra that are obtained. Another advantage is the extraction of morphological characteristic from the digital image.

There are some disadvantages in scanning electron microscopy. Since an SEM has dynamic optics, small amounts of contamination can accumulate on the conductive elements of the column. This contamination causes charges to build up on surfaces which result in beam deformation. The resultant beam is no longer circular but elliptical, resulting in an image with lower resolution. The SEM can, to a certain extent, compensate for this astigmatism (8).

Once the image is captured, the particle of interest is analyzed. This analysis is conducted using energy dispersive X-ray analysis. EDS is based on the interaction of a high energy source and the electrons within an atom. During these interactions inner shell electrons may be ejected and when a higher energy electron fills this hole, a photon in the X-ray range is emitted. All electrons in an atom revolve around the nucleus in orbitals. These orbitals may be clustered into shells which are named $\mathrm{K}, \mathrm{L}, \mathrm{M}, \mathrm{N} \ldots$ in order in increasing distance from the nucleus. The closer an electron is to the nucleus the greater the attraction between the electron and the nucleus. The closer a shell is to the nucleus the more tightly that shell is bound to the nucleus. Each element may be described by its electronic configuration. Copper, for instance, has an electronic configuration of $1 s^{2} 2 s^{2} 2 p^{6} 3 s^{2} 3 p^{6} 3 d^{7} 4 s^{2}$. In this element the $1 s^{2}$ electrons are in the $K$ 
shell, the $2 s^{2} 2 p^{6}$ electrons are in the L shell, the $3 s^{2} 3 p^{6} 3 d^{7}$ electrons are in the M shell, and the $4 \mathrm{~s}^{2}$ electrons are in the $\mathrm{N}$ shell (10). It is clear that each shell consists of a number of sub-shells.

When the electron hole occurs, the probability of it being filled by an electron from a specific higher energy level will determine the relative intensity of that transition. If an electron hole occurs in the K shell, as seen in Figure 2, the transitions from the L, $\mathrm{M}$, and $\mathrm{N}$ shells are termed $\mathrm{K} \alpha, \mathrm{K} \beta$, and $\mathrm{K} \gamma$ respectively. The line is named by the shell number in which the hole occurs. The subscript defines from which shell the transitioning electron originates. Each of these transitions can be further sub-divided based on which particular electron undergoes transition. For example, the Ka consists of the $K \alpha_{1}$ and $K \alpha_{2}$ transitions. It must also be noted that electron transitions can not simply occur from any higher to any lower orbital. These transitions obey the normal selection rules. The number and energy of the X-rays emitted from a specimen can be measured by using a spectrometer. For X-ray analysis in an SEM, both wavelength dispersive (WDS) and energy dispersive systems can be used. In this study an EDS system was used. The advantage of EDS over WDS is the speed of acquisition. The trade-off, however, is that the EDS system has lower resolution than that of the WDS system (11). An EDS system is unable to resolve the $K \alpha_{1}$ and $K \alpha_{2}$ transitions. The likelihood of observing a $\mathrm{K} \gamma$ is less than that of a $\mathrm{K} \beta$, which, in turn, is less than that of a $\mathrm{K} \alpha$. Using the combinations of the characteristic lines the elemental composition of a particular particle may be determined. 


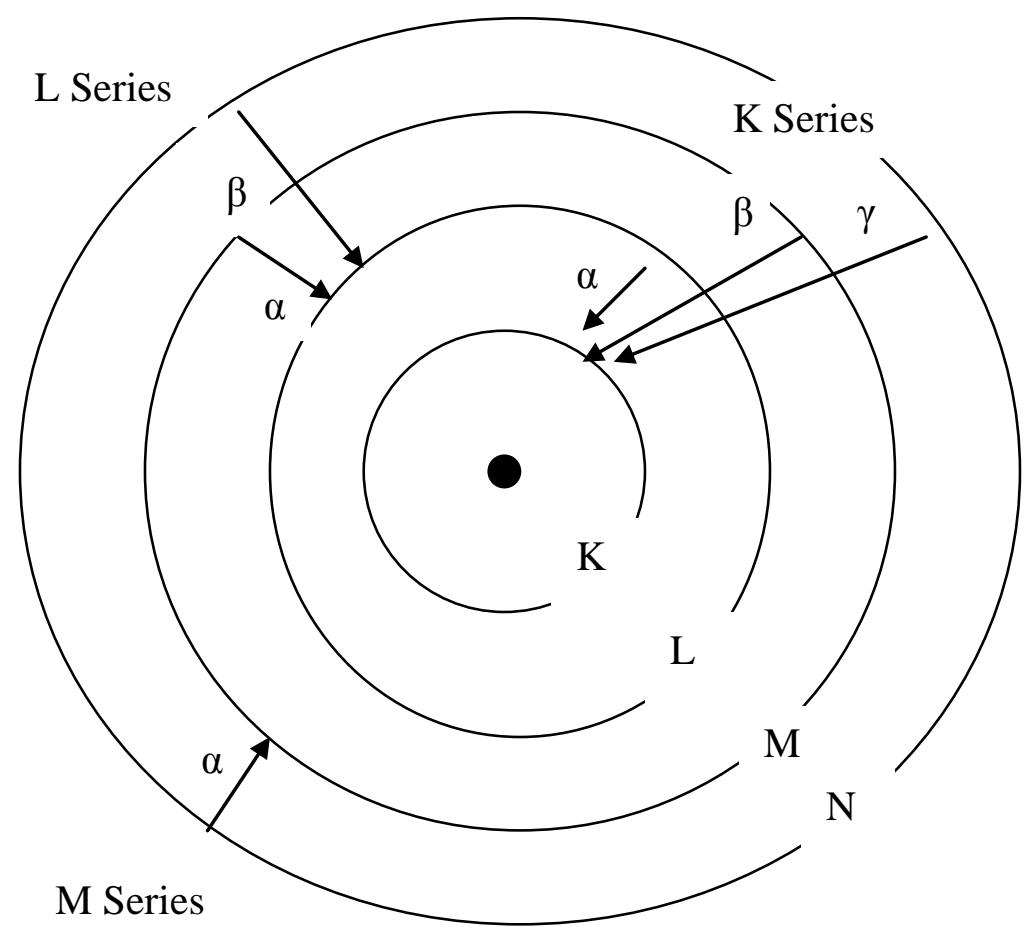

Figure 2: Diagram of electron energy levels and their respective series.

Although both the WDS and EDS systems use the characteristic X-rays generated by interactions with the individual elements to enable quantitative analyses, the limits of detection are different. A WDS system is able to detect elements down to trace levels when using spot sizes as small as a few micrometers. The primary advantage of WDS is its power to detect minor and trace elements due to its higher sensitivity and spectral resolution over EDS (11).

An EDS detector is designed in such a way that it can only process a single X-ray at a time. The detector system needs to determine the energy of the incoming X-ray and to count, using a multi-channel analyzer, the number of X-rays measured at that 
particular energy. Whilst the detector is processing the incoming signal, it is unable to process any other $\mathrm{X}$-rays at that time. Live time is defined as the time when the detector is not processing data and is available for analysis. Conversely, dead time is defined as the time involved in processing. Caution should be exercised during analyses with high dead times, since there is a high probability that a second event could interfere with the measurement of the first.

An SEM cannot measure the working distance to the specimen directly. The working distance is derived from the amount of current needed to drive the final lens such that the beam is focused on the surface of the specimen. The lenses in the SEM may build up lingering magnetism due to continual changes in the lens current. This magnetism will influence the computation of the working distance which results in incorrect magnification values. The selection of working distance for imaging and X-ray analysis is a trade-off (the best distance for imaging is not necessarily the best distance for EDS) (8). For imaging a short working distance provides the best resolution. For EDS analysis, the working distance needs to fulfill the geometry requirements of the electron beam, sample, and take-off angle of the detector.

There are many different techniques for the analysis of GSR. Each technique has its own advantages and disadvantages. All of these analytical techniques depend on the efficiency of the GSR collection at the crime scene or from the subject. It is very important to collect the evidence as soon as possible after the suspected shooting (1). For SEM/EDS analysis, suitable samples include most solids which are stable under a high vacuum. It is critical that the sample does not de-gas nor contain any fluids, since the high vacuum can cause damage to both the sample and the microscope. Examples 
include: metals, ceramics, polymers, and minerals. An EDS system if limited in both qualitative and quantitative detection of elements with low atomic numbers. This limitation is largely due to the window material of the detector. Depending on the detector type there is a limited detection of elements with a lower atomic number than sodium ( $\mathrm{Z}=11$ ) (12). Manufacturers have claimed to be able to detect beryllium $(\mathrm{Z}=4)$ (13). Suitable samples for the SEM/EDS analysis of GSR include adhesive stubs, tapes, clothing, skin, gauze and dry swabs subject to the vacuum constraints.

X-rays will not be generated unless the electron beam has sufficient energy to excite the elements of interest (8). When a sample is placed in the SEM it is bombarded by the beam of electrons. If a sample is nonconductive, then the accumulated electrons will cause the sample to charge up. To avoid the effects of charging, specimens are generally coated with a conductive medium, such as gold for high resolution imaging or evaporated carbon for X-ray analysis (11).

\subsection{Data Interpretation}

The analyst faces challenges in sample collection, analysis, and interpretation. In most cases, samples are collected by a crime scene examiner and forwarded to the forensic laboratory for analysis. It is also been discussed that the composition and a morphological criterion for unique and indicative particles is somewhat ambiguous. The common question of interpretation from the part of the investigator is whether the results of this analytical technique can determine who fired the weapon. This answer is not always clear. It requires a clear understanding of how the GSR was deposited and 
collected. Definite knowledge of the persistence and analysis of GSR is critical to its interpretation. It is the responsibility of the examiner to consider all of this information before forming any opinions. Even though GSR particles are composed of durable, heavy metals which can last in a normal environment, they may not persist indefinitely or remain on hands or clothing of a subject over time due to the physical activity of the subject. As with most forms of trace evidence, the finding that a specific type of particle was absent in a sample does not imply that that particle type was never present in or on the sample (1). Given the large dynamic magnification range of an SEM, it is possible to detect the small remaining particles of the hands of a suspect.

Moreover, an understanding of various factors, such as primer formulations and environmental sources of elements found in GSR is critical for the interpretation of analytical results. For example, since most primers contain lead, and primer residues are heterogeneous, some lead particles can be found on the hands of the shooter which originate from the primer (14). During the same discharge lead particles can be found on the hands of the shooter which have the origin in the fired bullet. For bulk analyses, many results are deemed inconclusive because the amounts of antimony, barium and lead are less than a prescribed threshold (15).

Populations of unique and indicative GSR particles and other lead-rich particles play a central role in forming an opinion of whether the presence of those particles is due to direct deposition or transfer due to casual contact (1). How will the fact that a suspect, who works with metals or compounds of antimony, barium, and lead, influence the outcome of the interpretation? How does the number of unique and/or indicative particles play a role in interpretation? 
Unique and indicative GSR particles can both found in samples from the discharge of a firearm. Unique particles are said to solely be the product of a firearm discharge whilst indicative particles are simply associated with the discharge. These indicative particles may well have come from the discharge of a firearm but they may also be the result of some other event. Thus indicative particles can not represent positive GSR and analysts should be cautious when an analysis yields multiple indicative particles and no unique particles.

During a study by Wolten et al. two particles which were found in a sample and which were consistent (indicative) with gunshot residue raised concern. The attribution of these particles to a firearm discharge was questioned on the grounds that they were associated with a larger number of very similar particles that were not considered to be gunshot residue. However, if these two particles were the only ones found, they would have been accepted as being consistent with gunshot residue (16). None of the occupational samples in this study were incorrectly identified by the analysts. The analysis of these samples by less experienced personnel may lead to difficulties in the interpretation of the results (16).

Each particle analyzed will be classified as unique, indicative, environmental, and unclassified using Table 1. If a particle which is classified as unique is found on a sample, then that sample will be classified as positive for gunshot residue.

The aim of this study is to evaluate the persistence of gunshot residue particles on the hands of a shooter. It is assumed that unique and indicative particles will be deposited on the hands of the shooter at the time of the discharge of a firearm. It is also assumed 
that some particles will also be deposited which originate from either the bullet or the primer but which can not be classified into the unique or indicative categories. Environmental particles are defined as those particles which may be found on the hands of an average person at any time during the day. Some particles in the environmental category may as have an origin in the discharge of a firearm but could also originate from other sources. Unclassified particles are particles which do not fall into one of the above categories. After the discharge of a firearm the number of unique and indicative particles on the hands of a shooter will decrease until none are left. The time that it takes for the complete loss to occur is a function of the activity of the individual. During this process there is an equilibrium of loss and gain of environmental particles. Particles in the unclassified category may be gained and lost according to the mechanisms of the unique/indicative and the environmental particles. The number of unique particles on a persons hand can be interpreted to be a concentration per unit area. The loss of these particles can possibly be describes by some decay function.

Persistence studies would answer the question of the maximum time it takes for all unique particles to be lost from the shooters hands. The implication of these data is the determination of a time frame beyond which the need for gunshot residue testing no longer exists (Figure 3). 


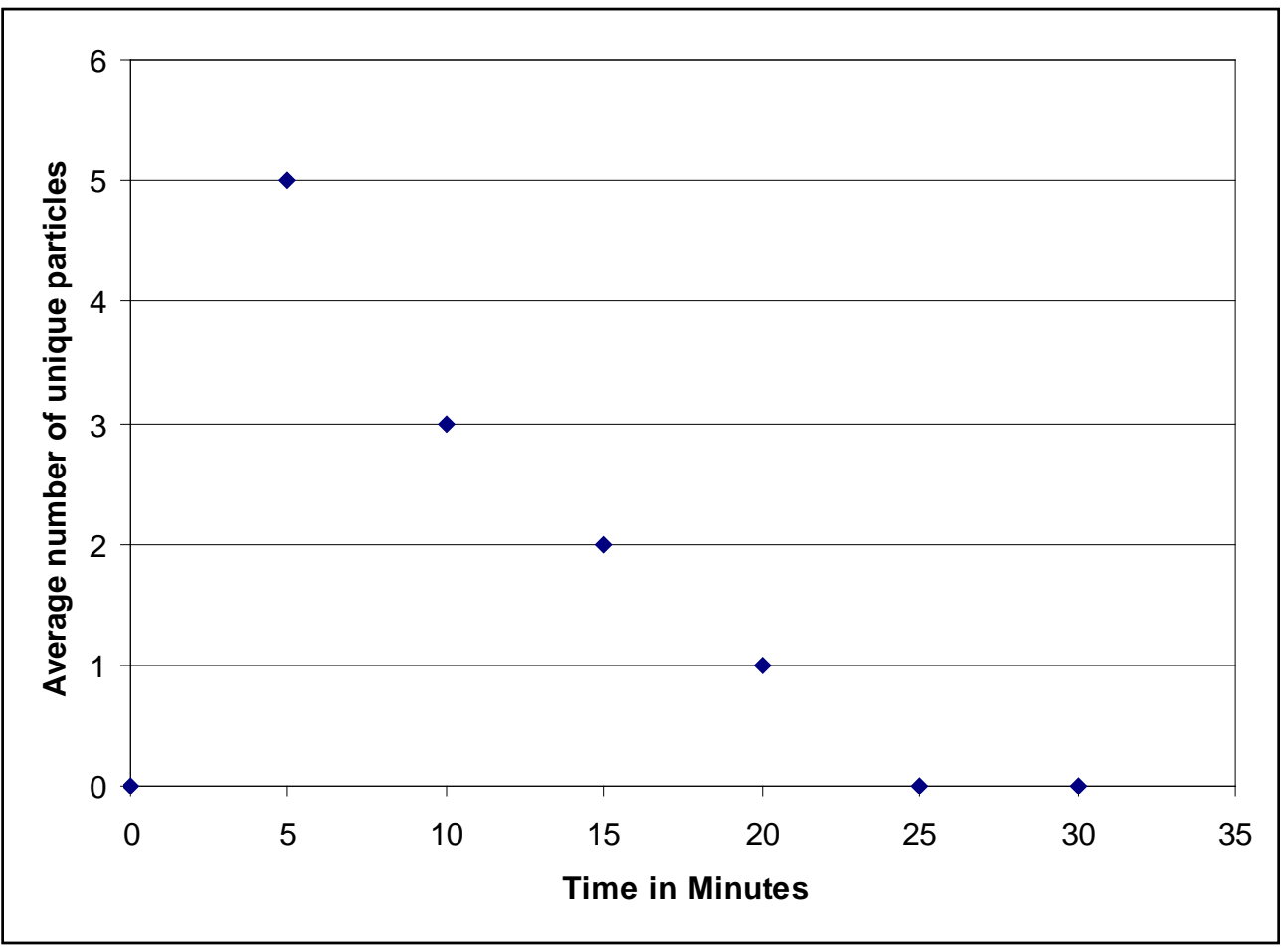

Figure 3: Example of persistence of unique gunshot residue particles

The rate of decay should be described by reaction kinetics. The concentration or number of particles on the hands of a shooter decreases over time. A question is whether this decay is a function of the initial concentration of GSR particles. In various studies it has been found that the number of particles deposited on the hands of the shooter varies even though the firearm and ammunition used have remained constant. It has also been shown that the firing of numerous shots does not affect the number of particles found on the shooters hand. If the loss of particles is described by zero or second order kinetics, then the rate is dependent upon the initial concentration. With first order kinetics the rate is independent of the initial concentration.

First order kinetics will be used to model the data from the persistence study. A first-order reaction depends on the concentration of only one reactant. 
Let $\mathrm{p}=[$ GSR particles $]$

$$
\begin{aligned}
& \text { Rate }=-\frac{\Delta p}{\Delta t}=k p \\
& \therefore-\frac{d p}{d t}=k p \\
& \text { thus } \\
& -\frac{d p}{p}=k d t
\end{aligned}
$$

Integrate the differential equation,

$$
\begin{aligned}
& -\int_{p_{0}}^{p_{t}} \frac{d p}{p}=k \int_{0}^{t} d t \\
& -\left\{\ln p_{t}-\ln p_{0}\right\}=k(t-0) \\
& \ln \frac{p_{t}}{p_{0}}=-k t \\
& \text { or, } \\
& \log \frac{p_{t}}{p_{0}}=\frac{-k t}{2.303}
\end{aligned}
$$

Where $\mathrm{p}_{0}$ is the number of GSR particles on the shooters hand directly after firing and $\mathrm{p}_{\mathrm{t}}$ is the number of GSR particles at some time $t$ after the discharge. The ratio of $\mathrm{p}_{t} / \mathrm{p}_{\mathrm{o}}$ is the fraction of particles remaining on the hands at time $t$. The rate of this reaction $-k t / 2.303$ is independent of the initial concentration. The integrated first-order rate law is given by:

$$
\ln [A]=-k t+\ln \left[A_{0}\right]
$$


This rate law is usually written in the form of the exponential decay equation:

$$
A=A_{0} e^{-k t}
$$

In a first-order process, the rate of decrease of a reactant is proportional to the amount of the reactant present $\left(\mathrm{A}_{0}\right)$. Therefore the kinetic constant must represent the fraction of the population of reactant present that will breakdown in a given time period and the fraction must be less than one. The analytical data will provide the number of particles present on the hands of the shooter at time $t$ after the shooting. The experimental data will be modeled using the exponential decay equation. A simplex optimization will calculate values for $\mathrm{A}_{0}$ and $k$ such that the difference of squares between the experimental and modeled data is minimized.

The half-life of a reaction is the time it takes for the reactant concentration to reach half of its initial value. This is the same as the radioactive decay of a radioactive nucleus (also a first order process). The determination of a half life for GSR particles will aid in the viability of an analysis of an aged GSR sample (17).

The half life of the GSR loss process will be calculated as follows:

Let $\quad \mathrm{p}_{\mathrm{t}}=$ number of particles at time $t$

$$
\mathrm{P}_{0}=\text { number of particles at time } 0
$$


For calculating the half life:

$$
\begin{aligned}
& p_{t}=\frac{1}{2} p_{0} \\
& \therefore \log \frac{\frac{1}{2} p_{0}}{p_{0}}=\frac{-k t_{1}}{2.303} \\
& \log \frac{1}{2}=\frac{-k t_{1}}{2.303} \\
& 0.301=\frac{-k t_{\frac{1}{2}}}{2.303} \\
& t_{\frac{1}{2}}=\frac{0.693}{k}=\frac{\ln 2}{k}
\end{aligned}
$$

The half-life is independent of $A_{0}$, therefore the initial number of GSR particles is irrelevant. The rate constant $\mathrm{k}$, and thus the half-life, should be the same for each independent firing sequence. 


\section{Chapter 2: Literature Review}

\subsection{Gunshot Residue formation and morphology}

GSR is formed from the primer, propellant, lubricant, and metals that are found in the bullet, bullet jacket, cartridge casing, and gun barrel. The first step in the firing of a bullet is the detonation of the primer. The primer is detonated when it is crushed by the force of the firing pin of the firearm. This drives hot gases and hot particles into the propellant and thereby ensuring ignition. Propellant in the cartridge case starts to burn, generating hot expanding gases. The ignition of the primer mixture and the propellant occurs in a matter of a few ten thousandths of a second. The high pressure from the heated gases forces the projectile out of the cartridge case into the barrel and out of the muzzle of the gun. The whole powder charge is never completely burnt. Particles of burnt and un-burnt powder and primer residues are propelled out of openings in the firearm and out of the barrel along with the projectile.

During discharge, revolvers and pistols leak residues from parts of the gun near the firing hand. There are three sources of deposit:

- Leakage from gaps in the firing mechanism. Revolvers have a small gap between the cylinder and the rear end of the barrel. Some gunshot residues are leaked from this gap and deposited on the firer during discharge.

- Emission at ejection of the cartridge case. In semi-automatic pistols, as the bullet moves out of the barrel, another mechanism opens the breech and ejects the cartridge. During this process, some gunshot residues are released and deposited. 
- Blowback from the muzzle cloud. The major portion of gunshot residues are emitted from the muzzle of the weapon and some of these residues may flash back and be deposited on the firer (2).

Krishnan (18) concluded that pistols and revolvers deposit more residues on the firing hand compared to the non-firing hand, but this may not always be the case because the concentration and/or location of the residue depends on the shooting stance and grip used. Generally for this type of weapon, the larger the caliber, the larger is the likely amount of gunshot residue to be deposited on the hands.

The gunshot residues on the firing hand are mostly deposited in the web area (19). This is the V-shaped part of the hand between the thumb and the index finger facing up the barrel of the firearm when the trigger is puller. This area is in closest contact to the gases escaping along the side and back of the gun during discharge. Residues may also be deposited on the forearm or sleeves and the front of the chest from both revolvers and pistols $(19,20)$. Mere handling or loading of a firearm, as opposed to firing, is inferred from the detection of residues on the palm and inside of the hand (19). 


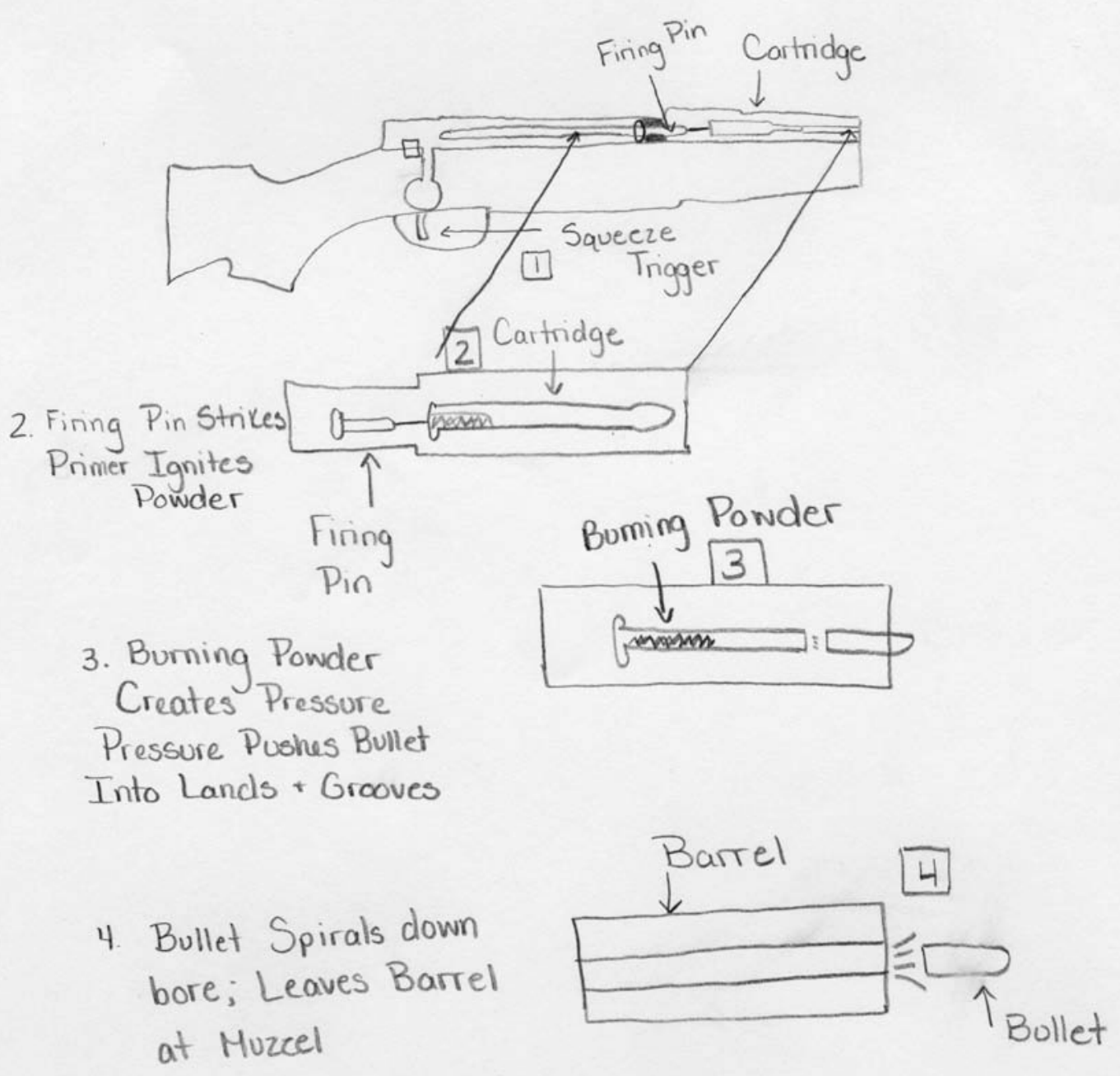

Figure 4: Representation of the firing cycle of a firearm (21)

The heat generated on ignition of the primer cause the inorganic ingredients of the primer mixture to vaporize. These vapors condense into droplets, which are further subjected to high pressure and temperature arising from the burning propellant powder.

The gunshot residues originating from the primer contain elements of primer compounds mainly lead, antimony, and barium (2). Understanding the origin and formation of GSR is essential to knowing the difference between authentic GSR and the 
environmental particles. Gunshot residue particles expelled from a firearm tend to have a uniform morphology and size. It is reported that in $70-100 \%$ of particles in a sample of gunshot residue are spheroidal (3). These spheroids may be stretched, dented, or otherwise distorted, but three-dimensional roundedness is a characteristic of this classification. The surfaces of the spheroids may be smooth or fuzzy, scaly, or covered with smaller spheres. Occasionally, they are capped, perforated, broken, or stemmed. A vast majority of the spheroidal particles have diameter of less then $5 \mu \mathrm{m}$ (3)

Particle morphology, when combined with the elemental composition, makes GSR quite distinct from many environmental particulates, including occupational particles such as lead aerosols, automobile exhaust, and consideration fumes that may contain one or more elements of GSR $(3,20)$.

\subsection{Color tests and bulk analysis}

In the past, criminalists relied solely on color reactions when examining items for deposits of GSR. These included the dermal nitrate test (22-25) and the Harrison and Gilroy tests for barium, antimony, and lead (22-26) The dermal nitrate test was introduced in 1933. This involved taking a cast of the back of the suspect's hand using hot paraffin wax. When cooled and set, the wax was peeled off along with embedded GSR particles. The cast was then sprayed with a $0.25 \%$ solution of $\mathrm{N}, \mathrm{N}-$ diphenylbenzidine $\left(\mathrm{C}_{24} \mathrm{H}_{2} \mathrm{ON}_{2}\right)$ in concentrated sulfuric acid. The reagent, like diphenylamine $\left(\mathrm{C}_{12} \mathrm{H}_{11} \mathrm{~N}\right)$ used later, gave a deep blue coloration with nitro-compounds from the partially burnt and unburned propellant particles $(14,23,27)$. While the test 
gave good information regarding the distribution of these particles, the test itself was only indicative for nitrates which are common in the environment. Oxidizers causing positive reaction are found in fertilizers, pharmaceuticals, fingernail polish, urine, and on hands of people after striking matches $(23,28)$. Although this method gave a positive reaction for nitrates, many other substances also gave a positive result for this test, thus making this method an unreliable indicator of GSR. In spite of the known limitations of this procedure, the use of diphenylamine tests to detect gunpowder residues continued because of the lack of other suitable test procedures to satisfy this need in criminal investigations (24).

In 1959, Harrison and Gilroy (26) demonstrated the presence of barium (Ba), antimony $(\mathrm{Sb})$, and lead $(\mathrm{Pb})$ in the firearm discharge residues and devised qualitative chemical tests to identify the presence of these elements (22-24). This test was called the Sodium Rhodizonate $\left(\mathrm{C}_{6} \mathrm{Na}_{2} \mathrm{O}_{6}\right)$ test. Swabs moistened with dilute hydrochloric acid (HCl) were used to collect the GSR on the hands. The swabs were dried and treated with triphenylmethylarsonium iodide. If antimony was present this reagent produced orange spots. The sample was dried again and after drying, a solution of sodium rhodizonate was added. This resulted in a red coloration with lead or barium. Upon adding dilute $\mathrm{HCl}$, the spots turned purple if lead was present (23). The advantage of this test over the dermal nitrate test was the low incidence of false positives. Although due to the limitation of sensitivity of colorimetric reactions used to detect $\mathrm{Pb}$, Sb, and $\mathrm{Ba}$, there was no widespread adoption of this method to replace the classical diphenylamine test. In spite of the low sensitivity of the diphenylamine test its use continued $(23,24)$. 
The search for a more definite and reliable means of detecting GSR led to the use of neutron activation analysis (NAA) (22-24, 29). In 1962, Ruch et al. presented the use of NAA for the identification of antimony and barium in GSR. At the time it was the most sensitive means of analyzing the majority of the elements (23). Ruch et al. used a $1 \%$ nitric acid $\left(\mathrm{HNO}_{3}\right)$ and filter paper removal technique and analyzed 130 samples to determine the control levels of $\mathrm{Ba}$ and $\mathrm{Sb}$ on hands (30). They found only 0.05-0.10 $\mu \mathrm{g}$ of Ba and 0.01-0.03 $\mu \mathrm{g}$ of Sb in most cases of controls (blank levels). Sampling hands for GSR after discharging a handgun resulted in values as high as $4 \mu \mathrm{g}$ of $\mathrm{Ba}$ and $1 \mu \mathrm{g}$ of Sb. This technique was not applicable to lead and analysis had to be conducted by trained personnel using a nuclear reactor as the neutron source. Despite these limitations, NAA has been extensively used to detect gunshot residues $(22,24,29)$ and to solve different problems in forensic science such as shooting distance determination and bullet identification (23).

During a study by Krishnan (18) the persistence of GSR was evaluated by firing more than 1500 test shots with fifty-seven different firearms, including pistols, revolvers, rifles, and shotguns. Krishnan stated that sample collection should be fast and provide minimal opportunities for contamination. Collection kits should be inexpensive, easily prepared, and tested for quality (specifically, low background levels of trace elements). Since quantitative results are required; the collection method should quantitatively and reproducibly remove the residues from the hands. Paraffin and other film lifts, swabs, washing, and tape lifts were used as sample collection methods. The results of the study indicated: 
- High concentrations of lead, barium, and antimony on the hands of a subject compared to hand blank levels indicate the presence of gunshot residue.

- The concept that there are higher concentrations of residues on the back of the hand of the firer while higher concentrations exist on the palm of a person who merely handled a gun is not always valid or reliable in case work.

- Residues persist on the hands longer in actual cases (up to $24 \mathrm{hr}$ in many instances) than indicated in laboratory experiments (approximately $2 \mathrm{hr}$ ).

- The technique is effective in detecting gunshot residue on hands. However, it is not always possible to determine how it got there. In exceptional cases where clearly one hand has more than the other and similar data are obtained by simulating the alleged incident (18).

In 1971, atomic absorption spectroscopy (AAS) as a method for the analysis of GSR was reported (31). Conventional flame AAS had sufficient sensitivity for detection of the levels of lead encountered in hand samples but was inadequate for barium and antimony.

Stone et al. (32) devised a series of experiments to determine the applicability of AAS, emission spectroscopy, and soft X-ray radiography for the analysis of gunshot residues. They found that each weapon differs in the amount of gunshot residues deposited on a firing hand or on clothing. Different residue depositions were found for revolvers, which depended on the individual chambering of the round. They seldom 
detected gunshot residues from semi-automatic pistols, unless in contact wounds or calibers less then .32.

According to Jones and Nesbitt (33) and Nesbitt et al. (34) a photoluminescence technique for GSR analysis was introduced. Samples were collected off of the back of each hand with a $2.5 \mathrm{~cm}$ disk coated on one side with Scotch no. 465 adhesive transfer tape. To prepare solutions for lead and antimony analysis, $0.5 \mathrm{ml}$ of $7 \mathrm{M} \mathrm{HCl}$ was pipetted onto the tape surface and allowed to soak and react for 3 min. After the HCl reaction, the acid sample was placed in a Suprasil quartz sample tube of precision bore. The tube was immersed into liquid nitrogen in a quartz optical Dewar flask and was analyzed for $\mathrm{Pb}$ and Sb content. The samples were excited in situ with a quartz-xenon arc lamp to detect the emission of $\mathrm{Pb}(385 \mathrm{~nm})$ and $\mathrm{Sb}(660 \mathrm{~nm})$. A tape-lift collection method (33), used in 168 test firings, was found to be superior to a wash procedure (32) for the collection of these GSR samples. Hand blank samples were collected from workers in a variety of occupations. Considerably more residues were detected in samples collected after an indoor firing than those from outdoors. Sb was detected in samples obtained after firing antimony free ammunition. The results of the persistence study indicated a concentration drop of about an order of magnitude after $1 \mathrm{hr}$. It was shown that GSR could be transferred from hand to hand and from hand to clothing, especially into trouser pockets. Similar results were obtained in 1975 by Kilty while determining antimony and barium concentrations by NAA (35).

Capillary electrophoresis (CE) has many attractive features such as a broad analytical spectrum, variety of separation modes and detection systems, high separation efficiency, mass-sensitivity, easy and inexpensive operation, and instrumental ruggedness 
(23). MacCrehan found residues of smokeless powder on the hands of a shooter using capillary electrophoresis (36).

\subsection{Particle Analysis using Scanning Electron Microscope}

Bulk analysis can detect trace amounts of lead, barium, and antimony in a sample, down to the nanogram range. The detection of these elements is therefore suggestive of GSR. However, this only proves that $\mathrm{Pb}, \mathrm{Ba}$, and $\mathrm{Sb}$ were present on the subject without correlating them to a particular source. In principle, each element could have originated from independent sources. These results, by nature, are non-specific. Scanning Electron Microscope (SEM) analysis, in contrast, analyzes on a particle-by-particle basis, thus retaining the individual feature characteristics lost in a bulk analysis. SEM/EDS can tie the presence of $\mathrm{Pb}, \mathrm{Ba}$, and $\mathrm{Sb}$ to a single, micron-ranged, generally spheroidal GSR particle.

Wolten et al. published a series of three seminal articles that present their major findings using the SEM/EDX method for GSR analysis. The first paper describes the nature of the residues from small arms cartridges as found on the hands of shooters, including occupational and environmental particles (3). The need to have a clear understanding of occupational residues in the interpretation of GSR was highlighted (37). Finally they discussed the application of the method to 100 cases performed for law enforcement agencies (16).

After characterizing GSR from a broad range of hand gun cartridges, the analytical procedure was found to be adequate for the analysis of casework. In favorable 
circumstances it was possible to limit the type of ammunition used by GSR analysis (3). In order to establish the prevalence of environmental $\mathrm{Pb}, \mathrm{Ba}$, and $\mathrm{Sb}$ particles 80 samples were collected from individuals with occupations such as automobile brake mechanics, lead smelters, lead-acid battery assemblers, auto mechanics, plumbers, and machinists. These samples were analyzed together with samples of actual discharges. The analyst had no background information of the origin of each sample. None of the occupational samples were falsely identified as GSR by these analysts. Less experienced analyst may encounter difficulties with these types of samples (37). A subject's occupation is relevant to the interpretation of the results of a GSR sample. Lastly, the third study examined overall case records where gunshot residue was used in determining the case. This included 86 cases that fall into one or another of the three categories: homicide/assaults, suicide/assaults, suicide/homicide decisions, and suicide verification. Gunshot residue was found in $90 \%$ of the cases that involved the use of handguns and in $84 \%$ of all cases (15).

The presence of GSR on an individuals hand indicates one of the following:

- The person fired a gun

- Handled a recently fired gun

- Was a close bystander at a shooting

The presence of paricles other than GSR can provide information about a subject's recent activities or environment, both in firearms-related and other types of cases (15).

The analysis of gunshot residue by SEM/EDS is considered to be the most reliable techniques for establishing whether a person had recently fired a gun $(38,39)$. It was the analytical method of choice in the pioneering studies of GSR deposition carried 
out by Basu et al (40). In a recent review of GSR analysis by Meng and Caddy (2), this perspective was reiterated. The methodology was described as potentially superior because it characterizes individual GSR particles via morphology and composition. A success rate of $79 \%$ for homicide/assault case has been cited as support for this technique $(2,41)$.

\subsection{Automated Search using Scanning Electron Microscope}

Automated systems have been developed to speed up GSR analysis by SEM/EDS. A number of time-saving approaches have been taken, such as the use of statistical considerations to reduce necessary specimens search area, and various residue collection and concentration techniques to improve collection efficiency and reduce levels of extraneous particulates. A further advance was based on the development of microprocessor controlled systems to aid in the automation of the GSR search and identification process. Such an automated system has four advantages over manual search procedure:

- Reduction of total search time and/ or increasing the search area on a sample

- $\mathrm{SEM} / \mathrm{EDS}$ operators are freed to perform other tasks

- Unattended automated runs (if performed at night then SEM/EDS instrumentation is available for other tasks during days)

- Reduction of human bias and error (for example, errors as a result of operator fatigue and distraction, and variability among different operators). 
It is clear that that the use of automated particle analysis system using SEM/EDS instrumentation could significantly improve the success of GSR searches and the confidence in search procedures and conclusions (42). Matricardi and Kilty stated that the automation of the search process is one of the essential developments needed before this technique will be used routinely (43).

Tillman conducted a study to evaluate an automated particle recognition and characterization program and its value for routine GSR particle analysis in forensic science laboratories (44). In this study, samples were collected using Scotch Brand 666 double-sided tape layered on 12-mm aluminum stubs. The stubs were dabbed over the back of the hand until no longer sticky. Immediately after collection the samples were carbon coated. Samples were collected immediately after firing and at intervals of 2, 4, and 6 hrs. Control samples were collected from individuals who had not discharged a firearm. Samples were analyzed using a Cambridge Stereoscan 200 scanning electron microscope with Particle Recognition and Characterization (PRC) program. Particle analysis in recent years has emerged as the most accurate and successful method of identifying and detecting GSR; (44) however, this advantage has been overshadowed by the often inordinate amount of time required for search and identification. Findings in this study support the effectiveness of using the PRC program for GSR identification (44). 


\subsection{Transfer and Persistence of gunshot residue}

Secondary transfer of GSR is always of concern to the analyst. Under normal circumstances when a suspected shooter is apprehended, GSR samples are collected. If these GSR kits contain adhesive- or tape-lift sampling devices, they may be analyzed using SEM/EDS (3, 16, 37, 43, 45, 46). While the detection of unique particles can provide confirmation of gunshot residue on a subject's hands, it cannot provide the mechanism of deposit. Although, handling and/or firing a gun is a primary means of deposition, transfer from a secondary source is possible $(43,46)$. Many studies have been performed to evaluate the risk of contamination of an individual by another individual, place, or object either before or during sample collection. Contamination of a previously collected sample is also of great concern.

According to Berk (4), a study to evaluate sources of secondary GSR contamination found that about $11 \%$ of the sampled police vehicles and detention facilities were found to contain three component GSR particles. These three component particles were obtained from table type surfaces (34 particles), restraining bars (20 particles), and tactical cars (2 particles). Although no studies were performed to evaluate the probability of transfer of these particles to the hand of an uninvolved individual, Berk concluded that the identification of GSR does not provide information on the mechanism of particle deposition. The low numbers of unique GSR particles detected in or on potentially contaminating sources suggests that the potential for secondary transfer is relatively low.

According to Gialamas (46), situations where police officers collect samples from suspects, the arresting officer's hands represent a theoretical source of GSR for secondary 
transfer. In this study 43 police officers from two departments were sampled at the conclusion of their shift. The officers were asked to refrain from washing their hands until collection had been completed. Information regarding the type of weapon they carried, last time fired, last time removed from holster, cleaned since firing, and last time hands were washed was also collected. Samples were analyzed for GSR using a CamScan Series IV SEM with a Robinson backscatter detector. The following particle classification scheme was used.

\begin{tabular}{|c|c|c|}
\hline Type & Elements & Morphology \\
\hline Unique & $\mathrm{Pb}, \mathrm{Sb}, \mathrm{Ba}$ & Spherical or non-spherical \\
\hline & $\mathrm{Ba}, \mathrm{Sb}$ & Spherical or non-spherical \\
\hline Consistent & $\mathrm{Pb}, \mathrm{Sb}$ & Spherical \\
\hline & $\mathrm{Pb}, \mathrm{Ba}$ & Spherical \\
\hline & $\mathrm{Ba}$ & Spherical \\
\hline & $\mathrm{Sb}$ & Spherical \\
\hline & $\mathrm{Pb}$ & Spherical \\
\hline Irregulars & $\mathrm{Pb}, \mathrm{Sb}$ & Non-spherical/ irregular \\
\hline & $\mathrm{Pb}, \mathrm{Ba}$ & Non-spherical/ irregular \\
\hline & $\mathrm{Ba}$ & Non-spherical/ irregular \\
\hline & $\mathrm{Sb}$ & Non-spherical/ irregular \\
\hline & $\mathrm{Pb}$ & Non-spherical/ irregular \\
\hline
\end{tabular}

Table 2: Classification scheme used in the Gialamas study

Of the 43 officers sampled, three (7\%) had unique GSR particles. In each of the samples from these three officers only one unique GSR particle was found. Twenty-five of the 43 officers (58\%) had no GSR particles, as indicated in Table 2, on their hands.

Considering that a police officer's firearm is carried, handled, and fired (whether officially or for practice) on a routine basis, it is suggestive that only a small fraction of the sampled officers had unique GSR particles on their hands (47). The low number of 
unique GSR particles suggests that the likelihood of secondary transfer to a subject will be small. It is impossible to determine whether the presence of particles on the officers' hands was through primary deposition or secondary transfer.

Several authors have stated that the loss of GSR is due to many reasons such as washing or rinsing hands, rubbing them against materials, putting them in pockets and handcuffing then behind the back $(16,29,35,45,47)$. The maximum time after which GSR was still detected varies significantly (1 hour (48), 2 hours (35), 3 hours (45, 49, 50), 4 hours (51), 12 hours (3, 16, 52), 17 hours (29), 24 hours (18), and 48 hours (26)). Several authors have reported that the number of GSR particles on the firing hand of a living person decreased rapidly over time (44, 45, 47, 51). Murdock (52) and Nesbitt et al. (34) observed that the largest loss of GSR took place within the first hour. Kilty found the amount of barium decreased by a factor of ten in the first hour of normal activity. Nesbitt et al. (48) reported a noticeable decrease of GSR after two and three hours. This study evaluated both the transfer and persistence of GSR particles.

In a different study, Nesbitt et al. (48) evaluated the effect of activity and elapsed time after shooting on the persistence of GSR on the back of the hands. In most cases the tests were carried out with one-round, one-hand, indoor firings of a .32 Llama pistol using 71-grain, fully jacketed bullets (Federal). The effect of placing hands in a pocket in the first persistence experiment, one round was fired with the .32 pistol and then the firing hand was placed into a front pants pocket three times. The back of the firing hand was then sampled with tape, and the tape samples were analyzed for $\mathrm{Pb}$ and Sb. Blank pocket samples, taken from pockets that had no previous contact with gunshot residue, were collected from the same persons whose pockets had been sampled during the 
persistence experiment. Tape adhesive mounted on 1-in. diameter sampling disks was pressed against the inside lip of the pockets to collect residues. Tests of the effect of normal activity on persistence of residue were made for one hand, indoor firings of the .32 pistol, which were followed by unrestricted activity for specified periods of time (except washing the hands was forbidden). The backs of both hands were sampled with tape following 1, 2, 3, and $4 \mathrm{hr}$ time delays (48). Representative hand blanks were examined in the SEM to establish a basis for differentiation of handblank particles from gunshot residue. Secondary electron micrographs and X-ray analyses of representative particles most easily confused with gunshot residue. The most frequently detected elements were silicon, iron, calcium, sulfur, chlorine, potassium, titanium, zinc, copper, and aluminum. Organic particles from the hand were abundant. Fortunately, their morphology is eagily recognized, and their X-ray fluorescence is weak; it consists mainly of bremsstrahlung from light elements. In an average handblank, there are also many mineral particles that contain silicon, calcium, or titanium. They have smooth surfaces with a crystalline appearance. Fibers are common; they contain primarily light elements and occasionally sulfur or chlorine. Particles from more than 20 handblanks were examined and none would be confused with gunshot residue. Automobile exhaust from leaded gasoline produces the particles that most resemble gunshot residue, particularly with respect to lead content and size. However, bromine was a prominent constituent of most of these contaminant panicles, whereas it is not detected in gunshot residue particles (48). All lead exhaust particles encountered had nondescript morphologies. Overall, 140 samples from hands that had fired were examined; only one displayed no evidence of residue. To test the success of identifying gunshot residue in a 
mixed group of residue specimens and handblanks, a blind test was carried out. Of 35 specimens, 17 were specimens collected immediately after firing and 18 were handblanks. Less than $1 \mathrm{~h} 3$ was required for the analysis of each specimen. The residue specimens were produced by single firings of .22 (10 of 17 firings), .38, $.380, .45$, and 9-ram caliber guns. Both revolvers and semiautomatics were used to obtain representative results. With the larger caliber semiautomatic and worn revolvers, particulate deposit was rich, and positive analyses required only brief searches for suitable particles. For sparse residue specimens produced by clean guns, such as the new .22 Ruger pistol, it was difficult to locate particles of interest, and a large number of environmental particles were subjected to X-ray analysis before a gunshot residue particle was identified.

A limited study (48) was made of the ability to detect residues collected from the hand 1, 2, and $3 \mathrm{~h}$ after firing. Subjects fired one round from a .22 caliber Colt revolver and then engaged in unrestricted activity, except that hand-washing and contact with sources of additional residue were avoided. Gunshot residue panicles were found on all but one of the 20 specimens. The negative specimen had been collected $2 \mathrm{~h}$ after firing, and the subject had engaged in vigorous activity. It was evident that a noticeable decrease in number of residue particles occurred for the 2 and $3 \mathrm{~h}$ samples.

In the Gialamas study (46) the largest decline in concentration of Sb was seen between the shooting and the $1 \mathrm{hr}$ sampling. In the case of $\mathrm{Pb}$, it was indistinguishable from background levels after 1 hour. Samples from the non-firing hand resulted in varying concentrations (five replicate shots). No Sb was detected at the $0 \mathrm{hr}$ and $4 \mathrm{hr}$ intervals. At the 1, 2, and $3 \mathrm{hr}$ intervals Sb was detected in two of the five samples. A 
.32 pistol was used to study the persistence of GSR. The Pb concentration was reduced to background levels at the $1 \mathrm{hr}$ mark for both the firing and non-firing hands. The average Sb concentration for the 1, 2, 3, and $4 \mathrm{hr}$ persistence intervals was $0.08,0.06$, 0.04 , and $0.03 \mu \mathrm{g}$, respectively. If the threshold value for $\mathrm{Sb}$ (attributed to a discharge) is set at 0.1 or $0.2 \mu \mathrm{g}$, all samples collected $1 \mathrm{hr}$ or more after firing will be judged negative (34)

Jalanti (47) conducted a replicate SEM/EDS study using a 9mm Luger pistol. For the first test shot samples were taken immediately, and then after 2, 4, and 6 hours for each test shot. Participants were instructed to continue their normal daily office activity without washing their hands. Samples were collected from the thumb, forefinger, and web area of the back and palm of each hand. Most GSR and Pb-rich particles were lost during the first two to four hours, confirming the observations of Murdock (49) and Nesbitt et al. $(48,52)$.

Fojtàšek et al. (7) evaluated the settling of GSR using various firearms. Different firearms were used with targets placed $2 \mathrm{~m}$ from the firearm and in the direction of 45 degrees to the right from the shooting distance. Petri dishes were used to collect particles which settled during given time periods. These dishes were in turn sampled by using a carbon taped stub. For a 9mm pistol the largest number of unique $\mathrm{PbBaSnSb}$ particles was found between 1.5 and 2.5 minutes after the discharge. For the $7.65 \mathrm{~mm}$ Browning, this time period was 4 to 5 minutes after the discharge. Finally for the .38 special, the the time period was from 4 to 8 minutes after the discharge. The authors concluded that it was not possible to determine a general time curve of GSR particle deposition. The deposition time depends upon the type of firearm and its caliber. Their experimental 
results suggest that contamination through settling of large GSR particles is limited to 8 minutes. For a revolver, this time period could be as long as 10 minutes. This is an extremely difficult experiment to design. Many factors, such as particle size, shape, density, environmental conditions, etc, will influence the results of this test. Furthermore, it is well described that the sampling of an individual directly after discharge yields a large number of GSR particles. It is unclear whether a particle which simply settles out of the atmosphere will be retained on the hands of an individual. 


\section{Chapter 3: Materials and Methods}

\subsection{Sample Collection}

In this study, shots were fired by four volunteers who were sampled for GSR at various times after the shooting. A $1 \mathrm{~cm}$ piece of carbon tape (Ted Pella Inc) was placed on a $1 \mathrm{~cm}$ aluminum stub (Electron Microscopy Sciences). The protective covering on the carbon tape was kept in place until sampling. The tape was trimmed to suit the diameter of the stub. The carbon stubs were dabbed on the back surfaces of the hand until they no longer retained their stickiness [Figure 5]. All collected stubs were stored in holders (Ted Pella, Inc.) to ensure no contact between stubs. The work area was thoroughly cleaned and sterilized prior to sampling.
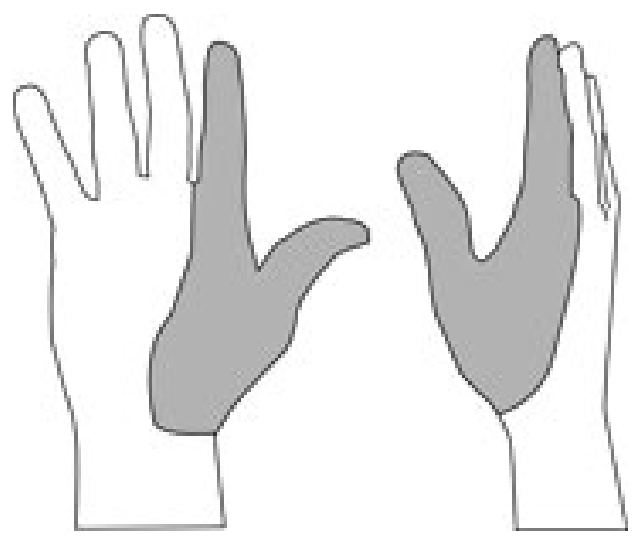

Figure 5: Area of the hands sampled by the carbon SEM stubs

The participants were asked to go about their everyday activities but refrain from washing their hands until after collection of the sample. The following flowchart indicates the procedure followed in this study [Figure 6]. 


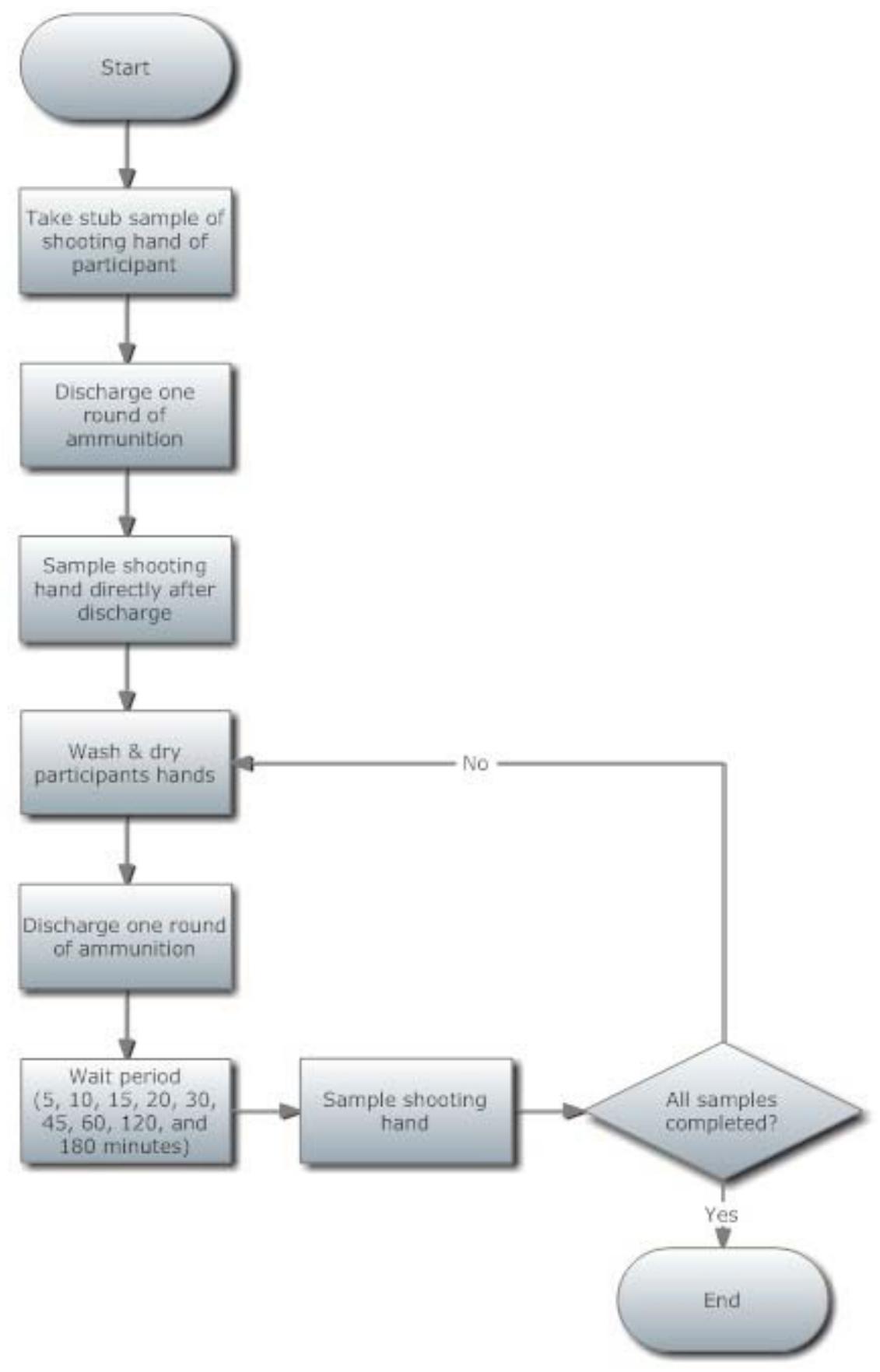

Figure 6: Collection method for the persistence of gunshot residue study

The collection of samples was repeated at 5, 10, 15, 20, 30, 45, 60, 120, and 180 minute time intervals. Samples were collected from each individual using two different 
firearms (Table 3). The cartridges used in the firearms were reloaded by the same individual using the same batch of primer, propellant, and bullet (Table 4). The same batch of primer, propellant, and bullet were used to reduce or eliminate factors that could influence the amount and nature of the GSR particles thus formed. Two different types of bullets were used, namely hollow point (HP) and full metal jacket (FMJ). Unique powder, manufactured by Alliant Powder in Radford, VA, was used as propellant.

\begin{tabular}{|c|c|c|}
\hline Manufacturer & Model & Caliber \\
\hline Springfield & xD M & $9 \mathrm{~mm}$ \\
\hline Smith \& Wesson & revolver & .38 \\
\hline
\end{tabular}

Table 3: Firearm Information

\begin{tabular}{|c|c|c|c|c|c|}
\hline Bullet type & Brand & Bullet & Alliant powder & Primer & Cartridge case \\
\hline HP & Reload MGB & 115 grains & 5.9 grains & CCI500 & brass \\
\hline HP & Reload MGB & 158 grains & 5.9 grains & CCI500 & brass \\
\hline FMJ & Reload MGB & 115 grains & 5.9 grains & CCI500 & brass \\
\hline FMJ & Reload MGB & 158 grains & 5.9 grains & CCI500 & brass \\
\hline
\end{tabular}

Table 4: Ammunition

\subsection{Sample Analysis}

The collected samples were analyzed for GSR using a JEOL JSM-6490LV

Scanning Electron Microscope (SEM) with a backscatter electron detector, and motorized stage (serial number MP14700049). Instrument operating conditions are listed in Table 5. 


\begin{tabular}{|ll|}
\hline \multicolumn{1}{|c|}{ Condition } & Setting \\
\hline Accelerating voltage & $20 \mathrm{kV}$ \\
\hline Working distance & $20 \mathrm{~mm}$ \\
\hline Specimen tilt & 0 degrees \\
\hline Magnification & $500 \mathrm{X}$ \\
\hline
\end{tabular}

Table 5: Operating conditions for the SEM/EDS

The EDS system is an Oxford Instruments INCA system. The software version is 4.09 with a system number of 12223 which includes an automated GSR detection system. The process of setting up the automated scan with the INCA system is a multi-step process taking into account its various parameters. These parameters include optimization, recipe setup, detection setup, spectrum setup, feature detection, and, running. A screen capture of this process is shown in Figure 7.

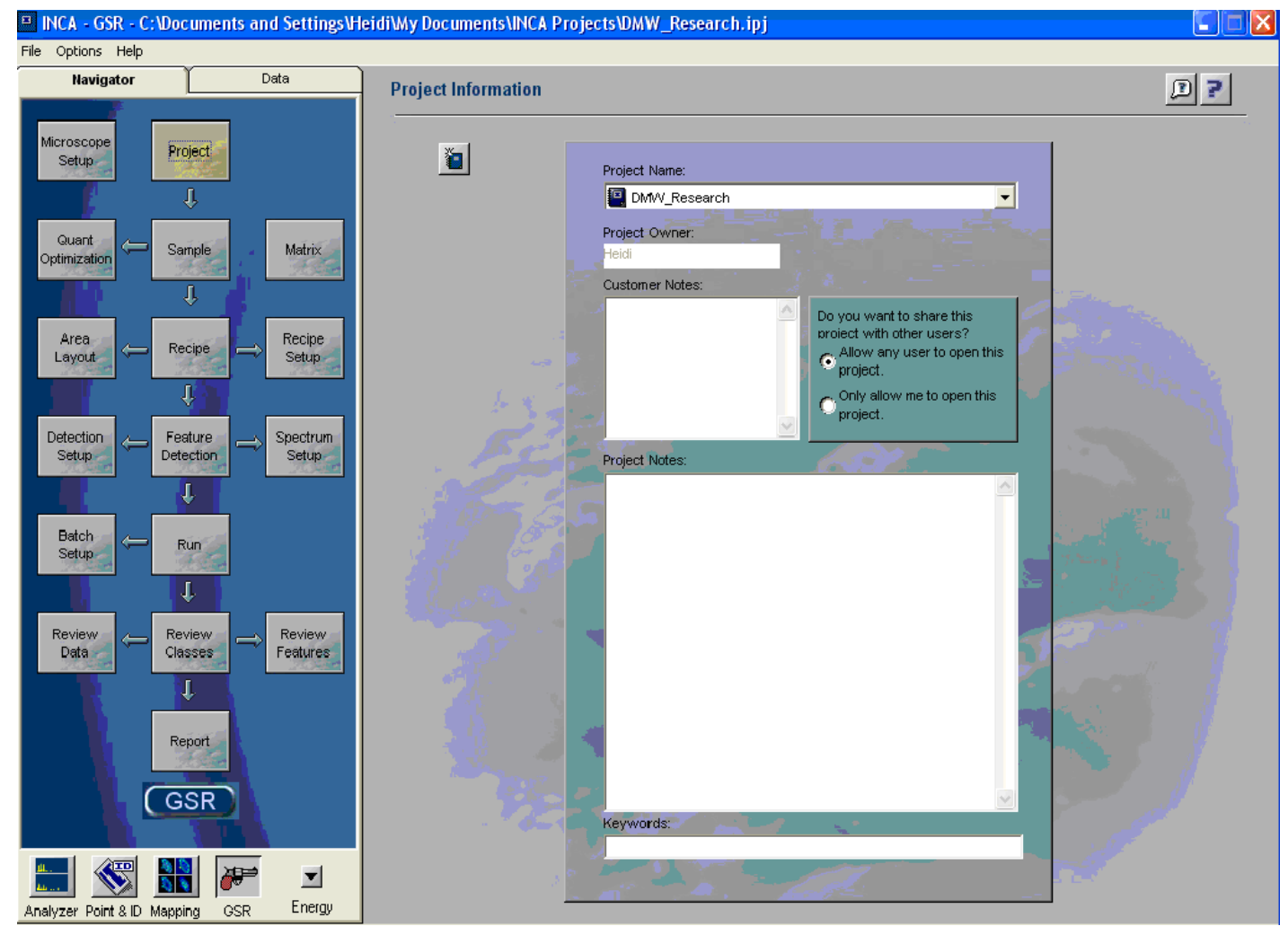

Figure 7: Automated scanning setup parameters 
Automation of the SEM/EDS gunshot residue analysis has been used in several laboratories nationwide $(42,44)$. This setup requires a project to be designed and each sample to be set up. A sample consists of 7 stubs. During the run the identity of each stub is documented and as well as its position in the sample holder. After samples are placed on the SEM a quantitative (quant) optimization is performed as shown in Figure 8.

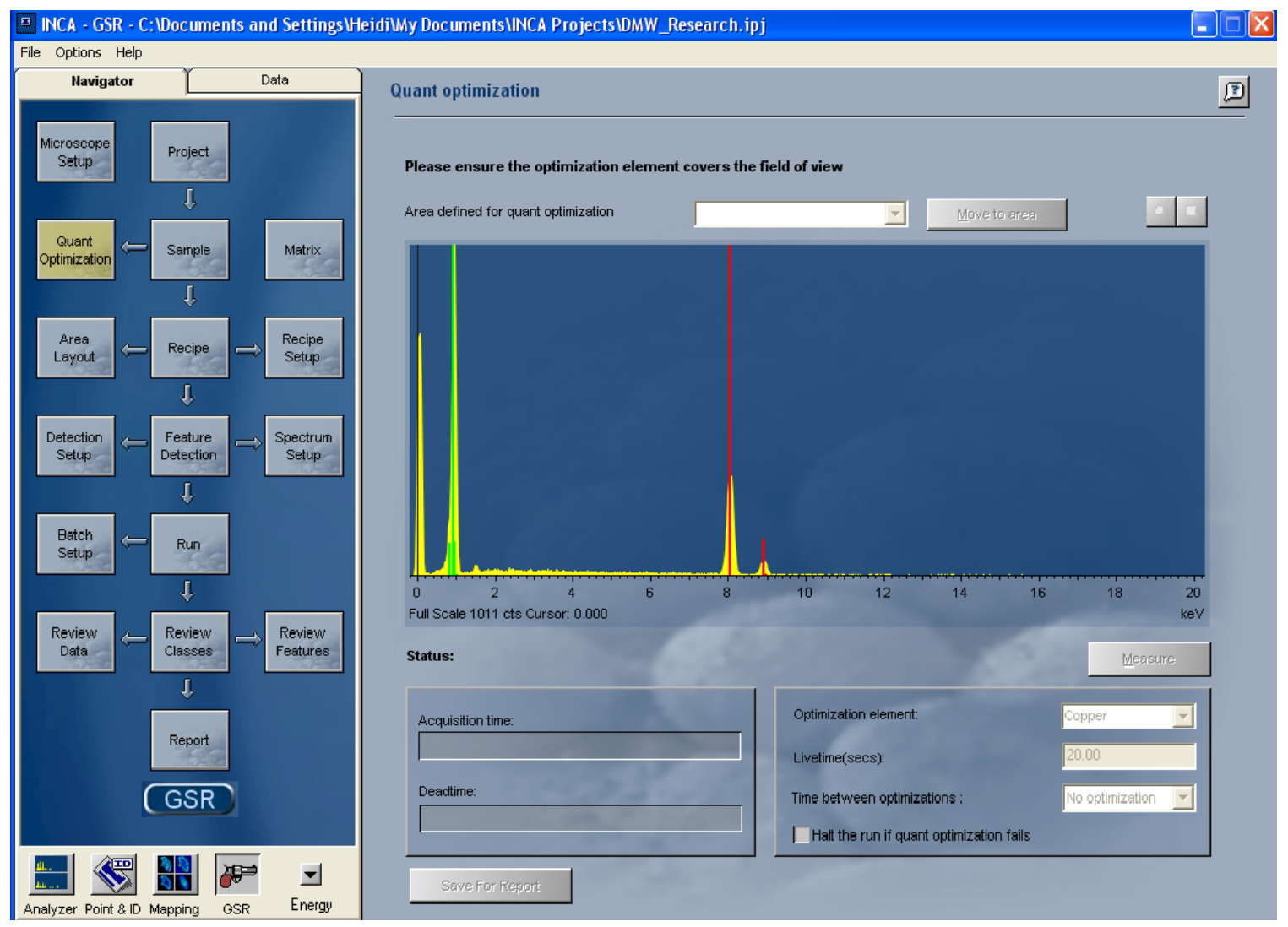

Figure 8: Quant Optimization step of the automated scan setup

Quantitative optimization for the automated searching system was achieved using a piece of copper plate which was placed on the sample holder. A piece of gold wire was also included as a standard. In Figure 8, copper was selected as the optimization element and a spectrum was collected. The peak heights and positions of the $\mathrm{Cu} \mathrm{K} \alpha$ and $\mathrm{K} \beta$ were 
evaluated to ensure optimal calibration. This step ensures that the peak height of the element exceeds the threshold required by the software for its identification.

For each analytical problem a "recipe” is designed to select the elements which are used to classify particles based on a stated classification scheme. A pre-programmed GSR “recipe” exsists within the INCA software. A variety of classification schemes exsist for GSR particles. Table 1 outlines the particle classification scheme used for the analysis of the gunshot residue using the GSR recipe.

After "recipe” selection, the "recipe” setup must be defined as shown in Figure 9.

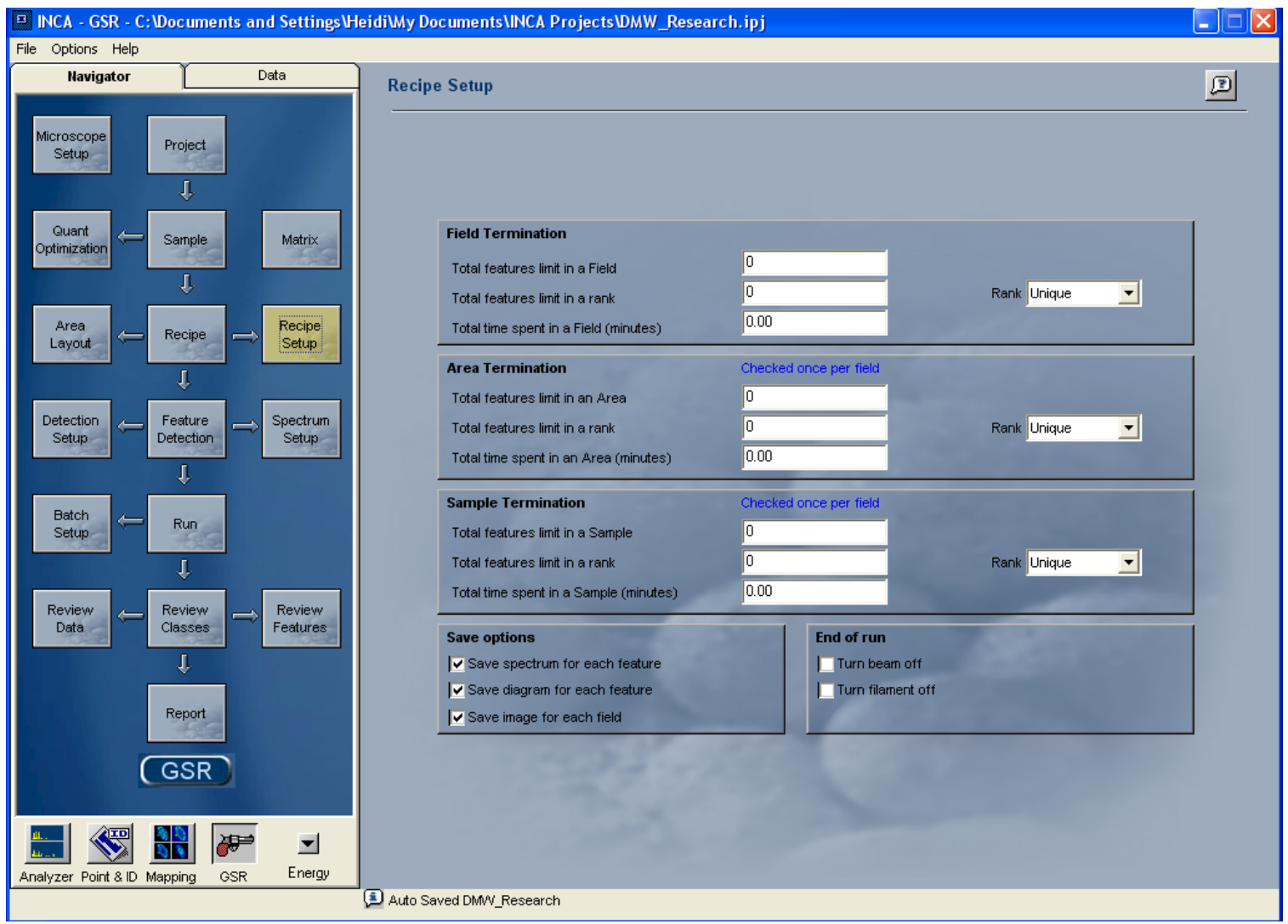

Figure 9: Recipe Setup screen

It is necessary to set termination parameters within the recipe setup. For these analyses a sample is defined as the set of 7 stubs, each stub is defined as an area, and 
each area is divided up into a number of fields. Termination parameters can be specified for each of these sub-divisions. These include the maximum number of features, the maximum number of features of a certain classification type, or the time spent within a specific sub-division. For this study, all termination parameters were left at zero because each stub needed to be analyzed fully to find the total number of particles. End of run options were selected to turn off the filament and beam after the run in order to prolong filament life.

An area layout has to be created to describe the seven circle stub holder in 3-D space within the SEM. The shape of the stub (circle) was selected and an image of the stub was captured through the SEM. Three reference points were selected around the image of the stub. Since the $\mathrm{x}, \mathrm{y}$, and $\mathrm{z}$ coordinates of the stage are associated with the image, the positions of the three reference points of the stub can be save in the area layout. This was done for all seven positions in the stub holder, and each position was labeled according to the number on the holder. Reference points to the $\mathrm{Cu}$ and $\mathrm{Au}$ were done in a similar manner except that they were defined as reference points in the area layout. These reference points are used as the starting position in the scan and are also used for the feature detection setup. Before every run the saved area layout is loaded and the stubs that need to be analyzed in that run have to be selected as shown in Figure 10 (a stub changes color from white to yellow when selected). 


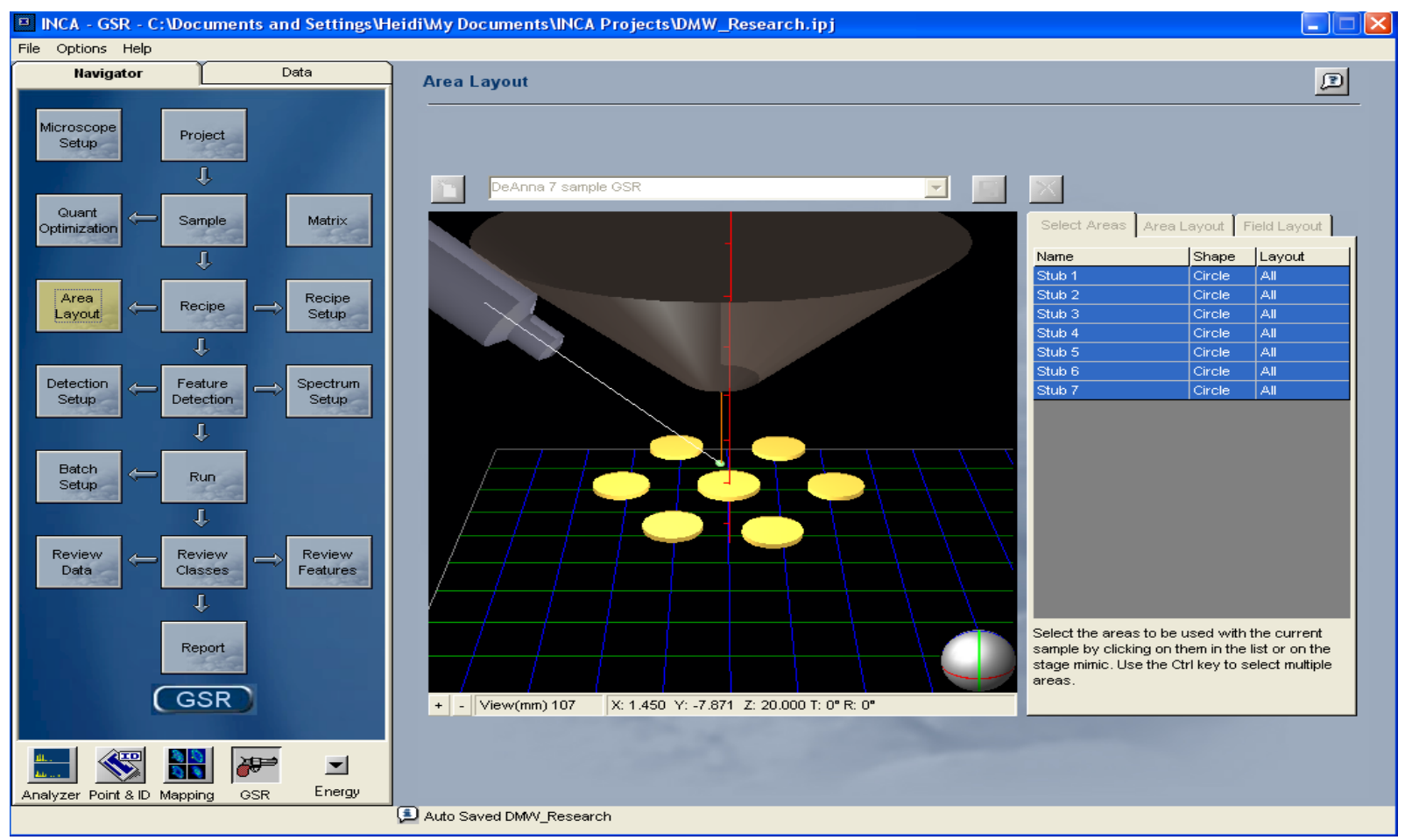

Figure 10: Area Layout screen with all seven stubs selected for analysis

The screen for the feature detection setup is shown in Figure 11. During feature detection, the grayscales are set for calibration and thresholding. Grayscale calibration uses the copper and gold samples as defined as references in the area layout. The sample holder is made out of aluminum (Al). When the backscattered image is acquired at the referent point it contains samples made up of $\mathrm{Al}(\mathrm{Z}=13), \mathrm{Cu}(\mathrm{Z}=29)$, and $\mathrm{Au}(\mathrm{Z}=79)$. The brightness and contrast of the SEM is then adjusted based on these three elements. For the analysis the average atomic number of the particles of interest is relatively high $(\mathrm{Pb}$ $(\mathrm{Z}=82)$, $\mathrm{Ba}(\mathrm{Z}=56)$, and $\mathrm{Sb}(\mathrm{Z}=51))$. The calibration of the image will allow for the determination of the average atomic number of an unknown particle based on its grayscale. The system provides the ability to adjust the calibration after a certain period of time to compensate for drift in the filament current. Filament drift usually results in 
increased contrast and brightness, the effect of which is to increase the number of candidate particles. The threshold selects the range of grayscales which can be related to particles of interest. When an image is collected of a field the threshold is applied and any particle which falls within the threshold becomes a candidate for EDS analysis. In Figure 11, any particle which has a grayscale between 26 and 161 will be a candidate for analysis.

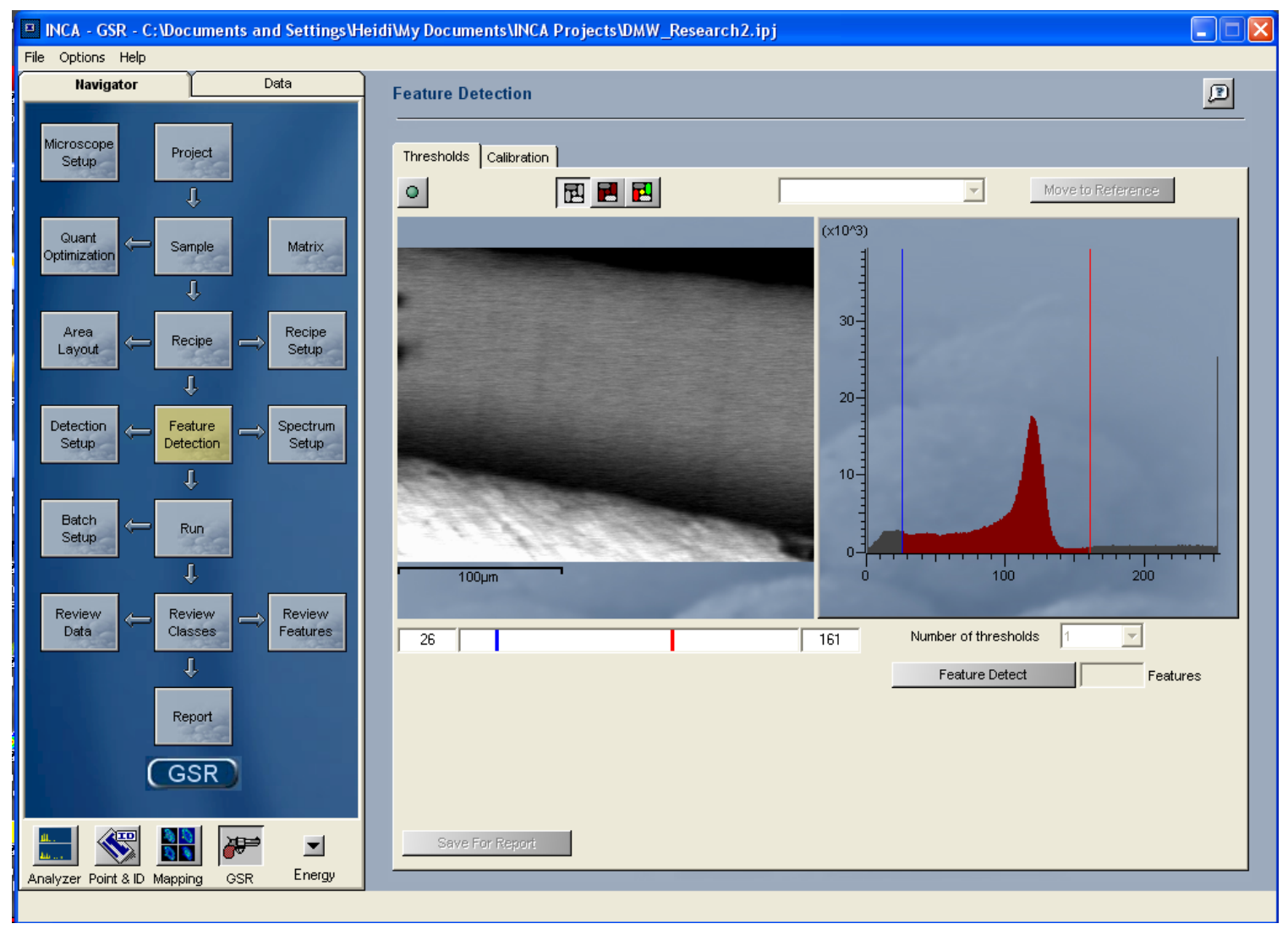

Figure 11: Feature detection screen to set threshold and calibration

Detection setup is used to specify field setup and is shown in Figure 12. Field setup is the resolution, signal, and time for first and second pass images. Increased resolution and time for each field has a large impact on the overall time for sample anaylsis. During this study, an intermediate field resolution of 1024 X 768 pixels was 
selected. The processing of a particle takes place in two steps. The first pass image is used to determine whether a particle is viable in terms of its grayscale. The time for the first pass image was set at $4 \mu$ s. The second image is used for processing and image analysis of the particles. This was set at $20 \mu$ s. These times were selected because a high quality image is not necessary for these processes and gave a good run time trade-off. The signal was set as SE (secondary electron). This is not an error but rather a foible of the software. The image type is set on the SEM and the image output is through a single channel to the INCA software. The INCA software channel is set only to SE.

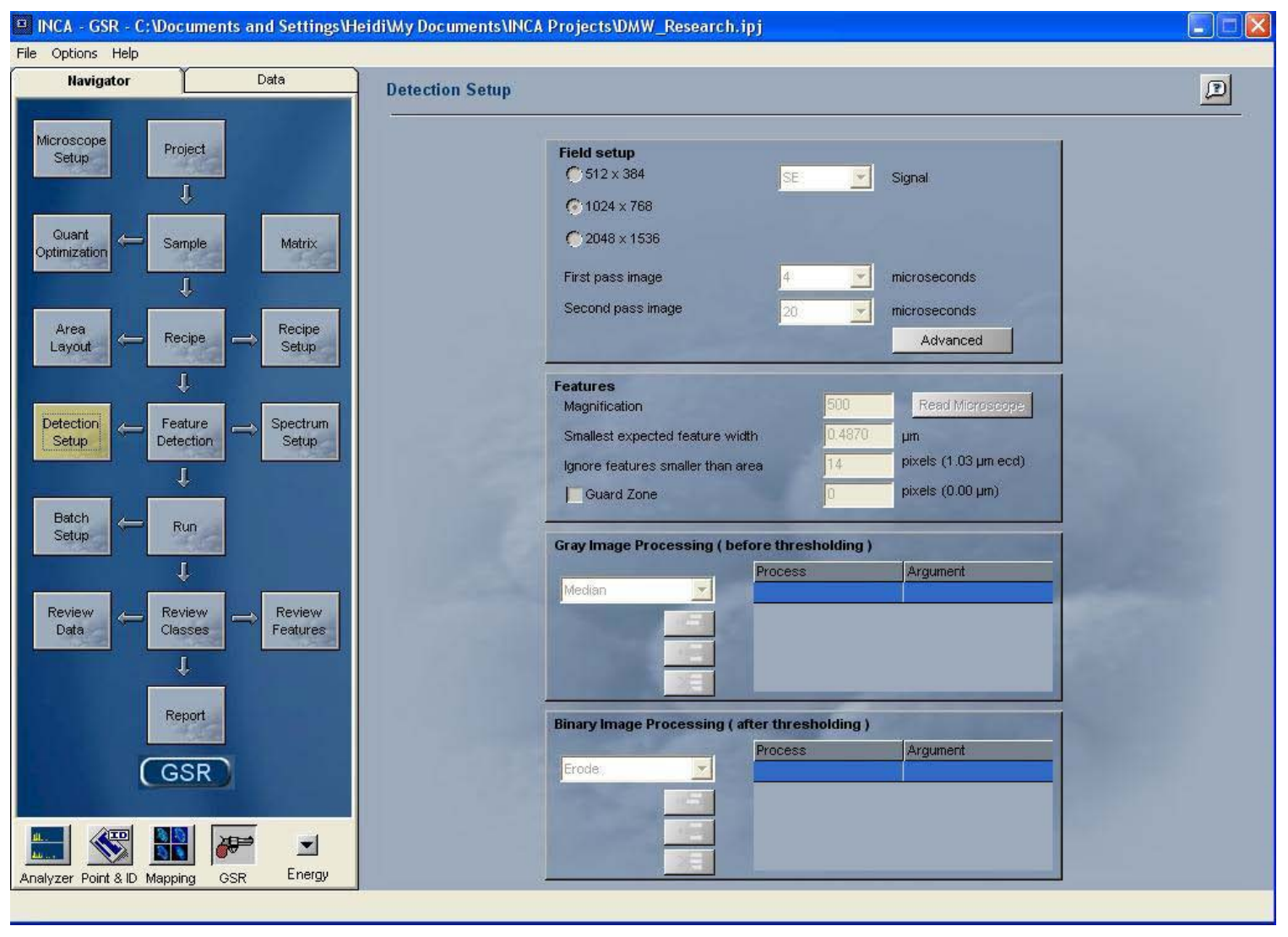

Figure 12: Detection setup used to set field and features 
Finally, spectrum setup is designed to specify the parameters of spectrum acquisition as shown in Figure 13. The spectrum can be acquired in one or two passes. During this study a single pass of three seconds (live time) was used. A process time of 3 was used. The greater the process times the better the signal to noise ratio but the longer the acquisition. Process times vary between 1 and 6 . Additionally, the spectrum range and channel resolution can be set.

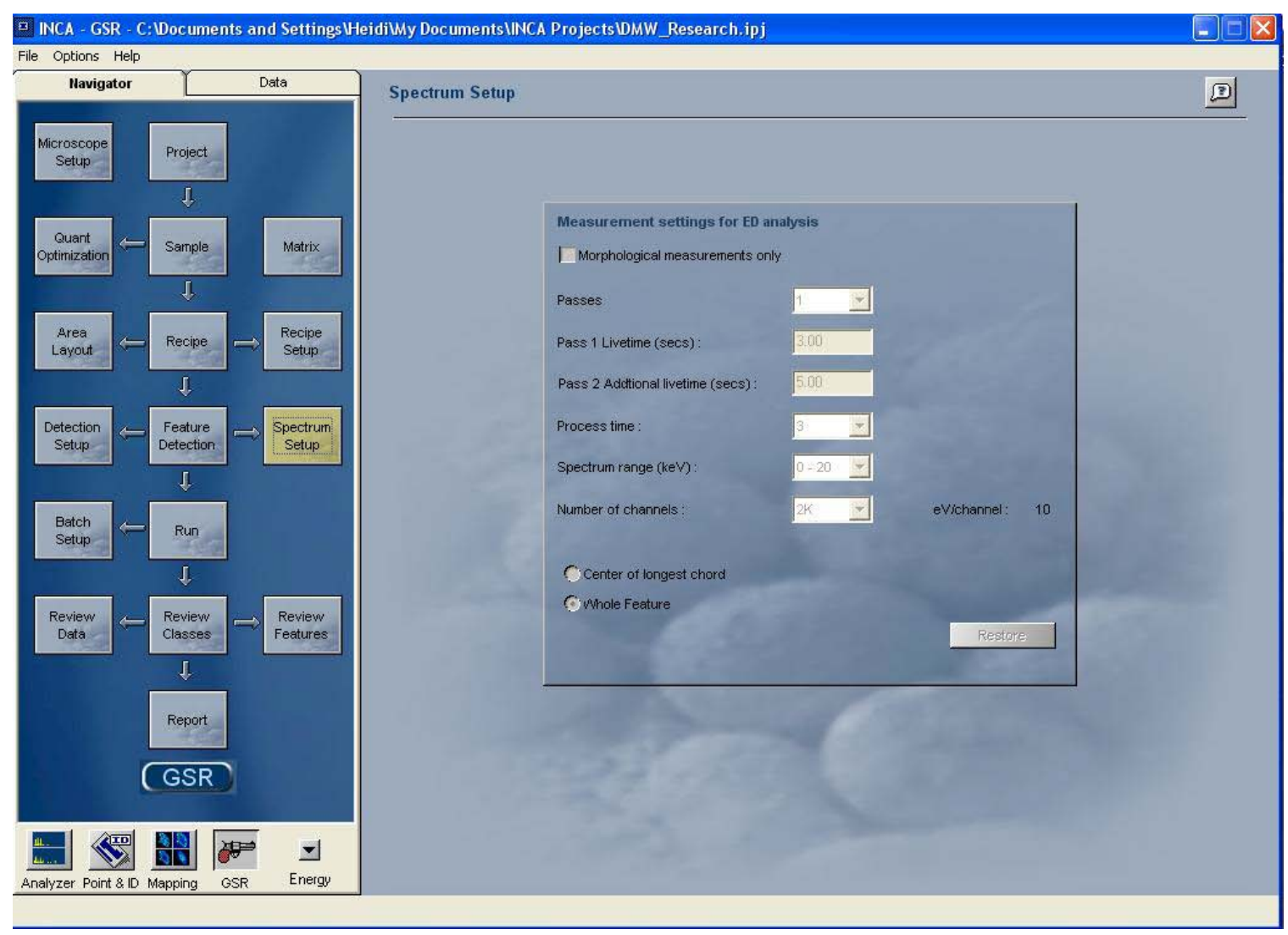

Figure 13: Spectrum setup used to time frames for spectrum analysis

After setting up this last parameter, the samples are now ready to run according to the setup. The run screen provides information regarding the run it proceeds (Figure 14). 


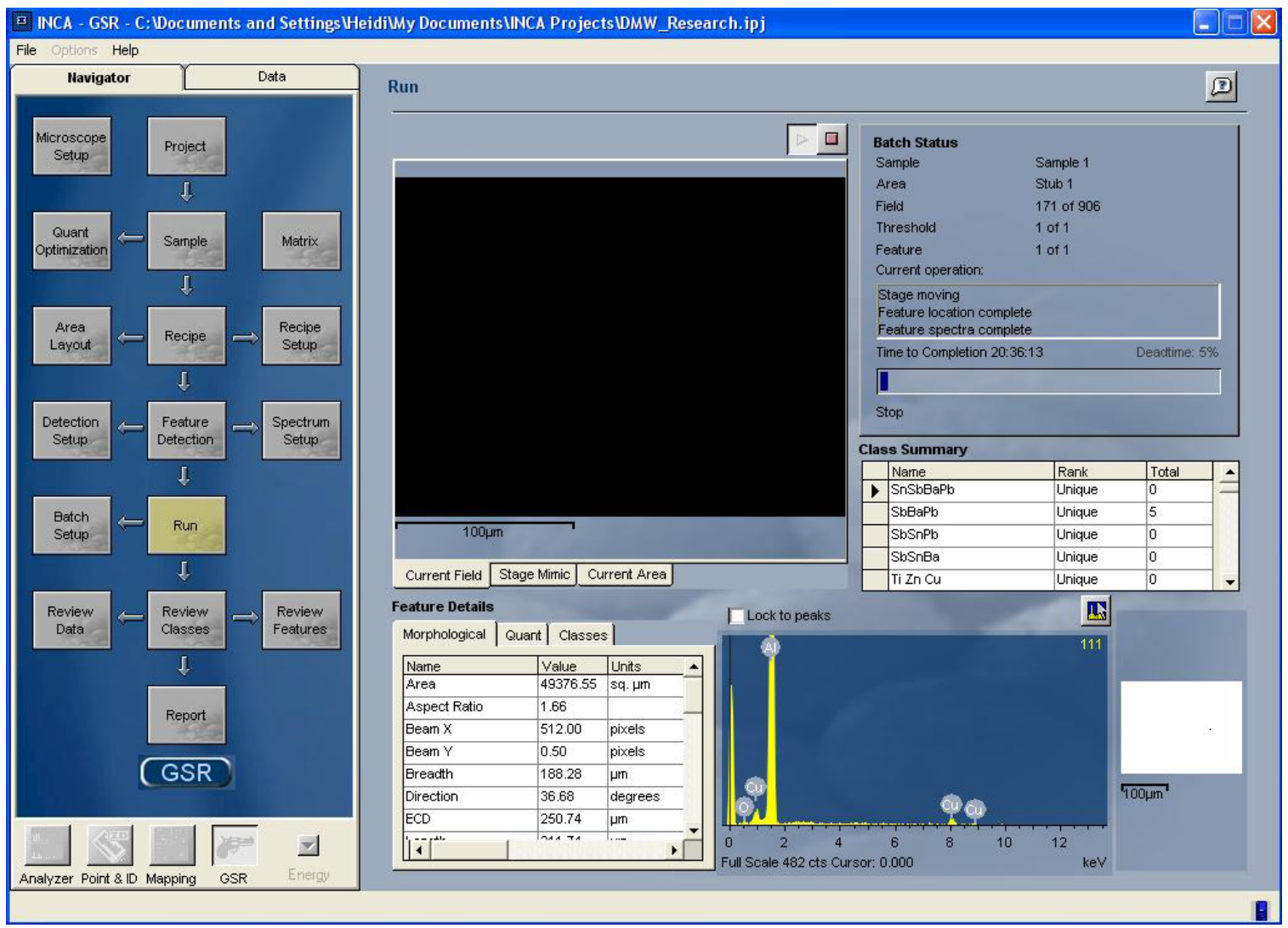

Figure 14: Run stage

The remaining screens are part of the data review process. The review classes screen displays the results of each stub and field (Figure 15). It is also possible to export all of these data to Microsoft ${ }^{\circledR}$ Excel spreadsheet for further analysis and review. Included in these data is information regarding each particle, its classification and a variety of morphological measures. 


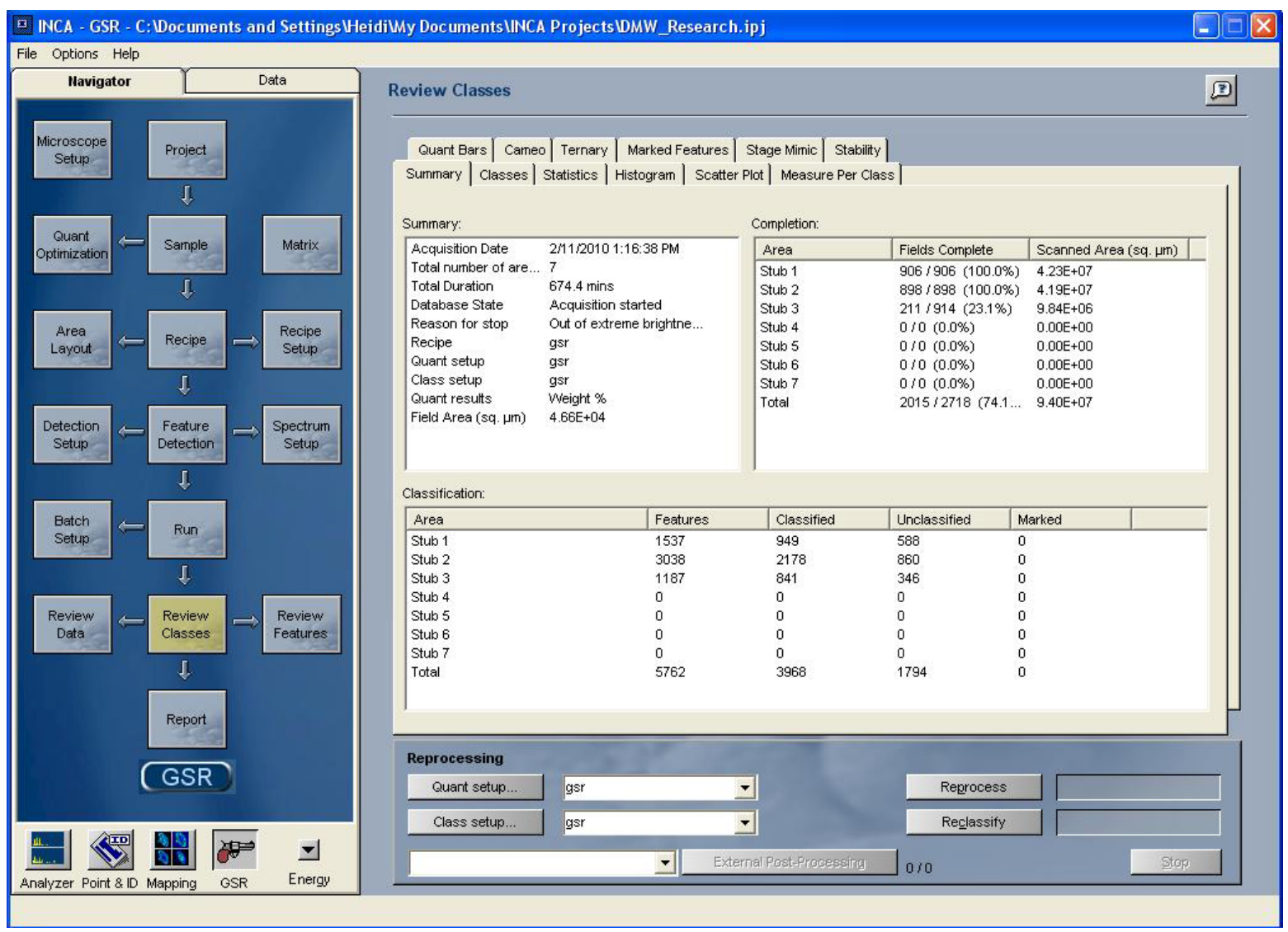

Figure 15: Review classes

\subsection{Revised Method}

The method was revised due to reoccurring issues with the automated scan.

Manual analysis used the same parameters as the automated scan. Scanning started in the upper left hand corner of the sample at a magnification of 700X. The stub was scanned from left to right, top to bottom. The brightness and contrast of the backscatter electron image was adjusted such that an iron (Fe) particle was just not visible. All bright particles that we encountered were analyzed. 


\section{Chapter 4: Results}

\subsection{Data from Manual Analysis}

At the start of the analysis, each stub collected needed to receive a sample number for identification. This sample number is the order in which it was analyzed on the SEM/EDS. The following two tables include this data (Table 6 \& 7).

\begin{tabular}{|c|c|c|}
\hline \multicolumn{3}{|c|}{ Participant 1-9mm } \\
\hline $\begin{array}{c}\text { Time between firing } \\
\text { and sampling (min) }\end{array}$ & $\begin{array}{l}\text { Stub } \\
\text { number }\end{array}$ & $\begin{array}{l}\text { Sample } \\
\text { Number }\end{array}$ \\
\hline Before contact & 5 & 1 \\
\hline 0 & 6 & 2 \\
\hline 5 & 7 & 3 \\
\hline 10 & 8 & 4 \\
\hline 15 & 9 & 5 \\
\hline 20 & 10 & 6 \\
\hline 30 & 11 & 7 \\
\hline 45 & 12 & 8 \\
\hline 60 & 13 & 9 \\
\hline 120 & 14 & 10 \\
\hline 180 & 15 & 11 \\
\hline
\end{tabular}

Table 6: Identification numbers for stub to sample analysis for participant 1

\begin{tabular}{|c|c|c|}
\hline \multicolumn{3}{|c|}{ Participant 3-9mm } \\
\hline $\begin{array}{c}\text { Time between firing } \\
\text { and sampling (min) }\end{array}$ & $\begin{array}{l}\text { Stub } \\
\text { number }\end{array}$ & $\begin{array}{c}\text { Sample } \\
\text { Number }\end{array}$ \\
\hline Before contact & 49 & 12 \\
\hline 0 & 50 & 13 \\
\hline 5 & 51 & 14 \\
\hline 10 & 52 & 15 \\
\hline 15 & 53 & 16 \\
\hline 20 & 54 & 17 \\
\hline 30 & 55 & 18 \\
\hline 45 & 56 & 19 \\
\hline 60 & 57 & 20 \\
\hline 120 & 58 & 21 \\
\hline 180 & 59 & 22 \\
\hline
\end{tabular}

Table 7: Identification numbers for stub to sample analysis for participant 3 
As each sample was analyzed every particle found was given a new site of interest on the sample. Each site of interest was classified as unique, indicative, environmental, or unclassified based on the elemental analysis and placed into an excel spreadsheet. All of the data that was received from the analysis of each sample is included in Appendix A. Analysis of the spreadsheet was done and a total number of particles per classification were gathered for each participant and then a total number of particles combined were found [Table 8, 9, and 10].

\begin{tabular}{|r|c|c|c|c|}
\hline & \multicolumn{4}{|c|}{ Classification of particles from Participant 1 } \\
\hline & Unique & Indicative & Environmental & Unclassified \\
\hline Before Contact & 1 & 0 & 7 & 1 \\
\hline 0 & 20 & 15 & 41 & 67 \\
\hline 5 & 19 & 7 & 11 & 3 \\
\hline 10 & 12 & 4 & 21 & 6 \\
\hline 15 & 12 & 10 & 18 & 4 \\
\hline 20 & 10 & 13 & 61 & 23 \\
\hline 30 & 5 & 8 & 35 & 18 \\
\hline 45 & 1 & 2 & 33 & 21 \\
\hline 60 & 0 & 0 & 5 & 2 \\
\hline 120 & 0 & 0 & 20 & 2 \\
\hline 180 & 0 & 0 & 4 & 0 \\
\hline
\end{tabular}

Table 8: Participant 1 number of particles for each classification at each interval

\begin{tabular}{|r|c|c|c|c|}
\hline & \multicolumn{4}{|c|}{ Classification of particles from Participant 3 } \\
\hline & Unique & Indicative & Environmental & Unclassified \\
\hline Before Contact & 2 & 1 & 13 & 9 \\
\hline 0 & 126 & 30 & 19 & 31 \\
\hline 5 & 19 & 8 & 38 & 13 \\
\hline 10 & 19 & 16 & 40 & 18 \\
\hline 15 & 8 & 7 & 28 & 12 \\
\hline 20 & 7 & 17 & 32 & 15 \\
\hline 30 & 0 & 0 & 1 & 26 \\
\hline 45 & 2 & 0 & 9 & 2 \\
\hline 60 & 1 & 1 & 10 & 11 \\
\hline 120 & 1 & 0 & 7 & 5 \\
\hline 180 & 0 & 1 & 4 & 2 \\
\hline
\end{tabular}

Table 9: Participant 3 number of particles for each classification at each interval 


\begin{tabular}{|c|c|c|c|c|}
\hline & \multicolumn{4}{|c|}{ Classification of particles total } \\
\hline & Unique & Indicative & Environmental & Unclassified \\
\hline $\begin{array}{l}\text { Before } \\
\text { Contact }\end{array}$ & 3 & 1 & 20 & 10 \\
\hline 0 & 146 & 45 & 60 & 98 \\
\hline 5 & 38 & 14 & 81 & 35 \\
\hline 10 & 31 & 20 & 61 & 24 \\
\hline 15 & 20 & 31 & 163 & 63 \\
\hline 20 & 17 & 30 & 69 & 38 \\
\hline 30 & 5 & 8 & 36 & 44 \\
\hline 45 & 3 & 2 & 42 & 23 \\
\hline 60 & 1 & 1 & 15 & 13 \\
\hline 120 & 1 & 0 & 27 & 7 \\
\hline 180 & 0 & 1 & 8 & 2 \\
\hline
\end{tabular}

Table 10: Total number of particles for each classification at each interval

The presence of unique and perhaps of the indicative particles is somewhat puzzling. In some instances the participants did not wash their hands before the intial shot. Two of the four participants are very active shooters.

\subsection{Results from manual analysis}

The particles identified on each stub were classified according the the classification scheme. For the unique and indicative classes the decrease in the number of particles was modeled using first order kinetics. The experimental data were plotted in Microsoft Excel and initial estimates for $\mathrm{k}$ and $\mathrm{A}_{0}$ were made. A plot of the first order kinetic decay curve was also included. The square of the difference between the observed and calculated number of particles was included in the spreadsheet. The sum of these differences was placed in the target cell. Using the solver function the target cell 
(sum of the difference of the squares) was minimized by adjusting the values for $\mathrm{k}$ and $A_{0}$. To ensure that the values were not of a local minimum a number of differing estimates of $k$ and $A_{0}$ were used.

\begin{tabular}{|llll|}
\hline$k$ & 0.05 & & \\
A0 & 21.4 & half life & 15.2 \\
\hline
\end{tabular}

\begin{tabular}{|r|c|r|r|}
\hline \multicolumn{1}{|c|}{$\mathbf{t}$} & A & A calc & \multicolumn{1}{c|}{ dos } \\
\hline $\begin{array}{l}\text { Before } \\
\text { Contact }\end{array}$ & 1 & & \\
\hline 0.00 & 20.00 & 21.37 & 1.88 \\
\hline 5.00 & 19.00 & 17.01 & 3.96 \\
\hline 10.00 & 12.00 & 13.54 & 2.37 \\
\hline 15.00 & 12.00 & 10.78 & 1.49 \\
\hline 20.00 & 10.00 & 8.58 & 2.02 \\
\hline 30.00 & 5.00 & 5.44 & 0.19 \\
\hline 45.00 & 1.00 & 2.74 & 3.03 \\
\hline 60.00 & 0.00 & 1.38 & 1.91 \\
\hline 120.00 & 0.00 & 0.09 & 0.01 \\
\hline 180.00 & 0.00 & 0.01 & 0.00 \\
\hline & & & \\
\hline & & Sum= & 16.9 \\
\hline
\end{tabular}

Table 11: Calculated data from Excel for Participant 1 using first order kinetics for unique GSR particles 


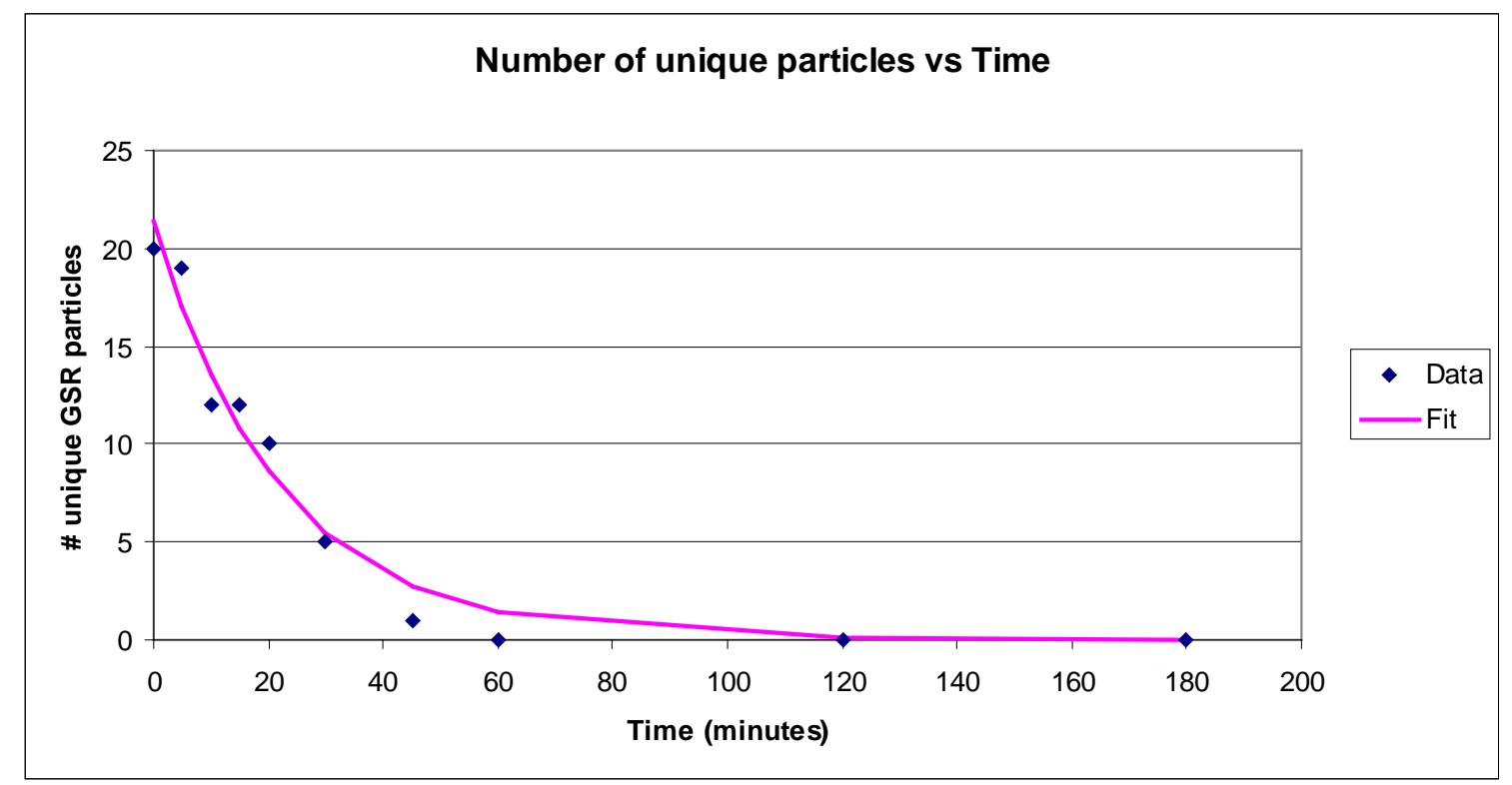

Figure 16: Number of unique GSR particles vs. time for participant 1

As seen in Table 11 the goodness of fit can be measured by the sum of the difference of squares (in this case 16.9). The results indicated $\mathrm{A}_{0}$ of 21.4. Given the type of experiment the value of $\mathrm{A}_{0}$ should be an interger, but for the understanding of the mechanism of loss the possibility of a real value for $\mathrm{A}_{0}$ is accepted. The advantage of first order kinetics is the independence of the initial concentration. The rate constant for this experiment was estimated to be 0.05 which convert to a half-life of about 15.2 minutes. This half-life implies a relative concentration of GSR particles of $6 \%$ of the original concentration after one hour. This is in agreement with some of the previous published data. Figure 16 illustrates these data graphically. The experimental data are represented by the blue diamonds whilst the pink line represents the fit line from first order kinetics. Based on this the data obtained from the study follows a good representation of the first order decay model. 


\begin{tabular}{|llll|}
\hline$k$ & 0.3164259 & & \\
$\mathrm{AO}$ & 125.26451 & half life & 2.190551 \\
\hline
\end{tabular}

\begin{tabular}{|r|r|r|c|}
\hline $\mathbf{t}$ & \multicolumn{1}{|c|}{ A } & A calc & dos \\
\hline 0 & 126 & 125.2645 & 0.540945 \\
\hline 5 & 19 & 25.74648 & 45.51499 \\
\hline 10 & 19 & 5.291852 & 187.9133 \\
\hline 15 & 8 & 1.087671 & 47.78029 \\
\hline 20 & 7 & 0.223557 & 45.92019 \\
\hline 30 & 0 & 0.009444 & $8.92 \mathrm{E}-05$ \\
\hline 45 & 2 & $8.2 \mathrm{E}-05$ & 3.999672 \\
\hline 60 & 1 & $7.12 \mathrm{E}-07$ & 0.999999 \\
\hline 120 & 1 & $4.05 \mathrm{E}-15$ & 1 \\
\hline 180 & 0 & $2.3 \mathrm{E}-23$ & $5.29 \mathrm{E}-46$ \\
\hline & & & \\
\hline & & & 333.6695 \\
\hline
\end{tabular}

Table 12: Calculated data from Excel for Participant 3 using first order kinetics for unique GSR particles

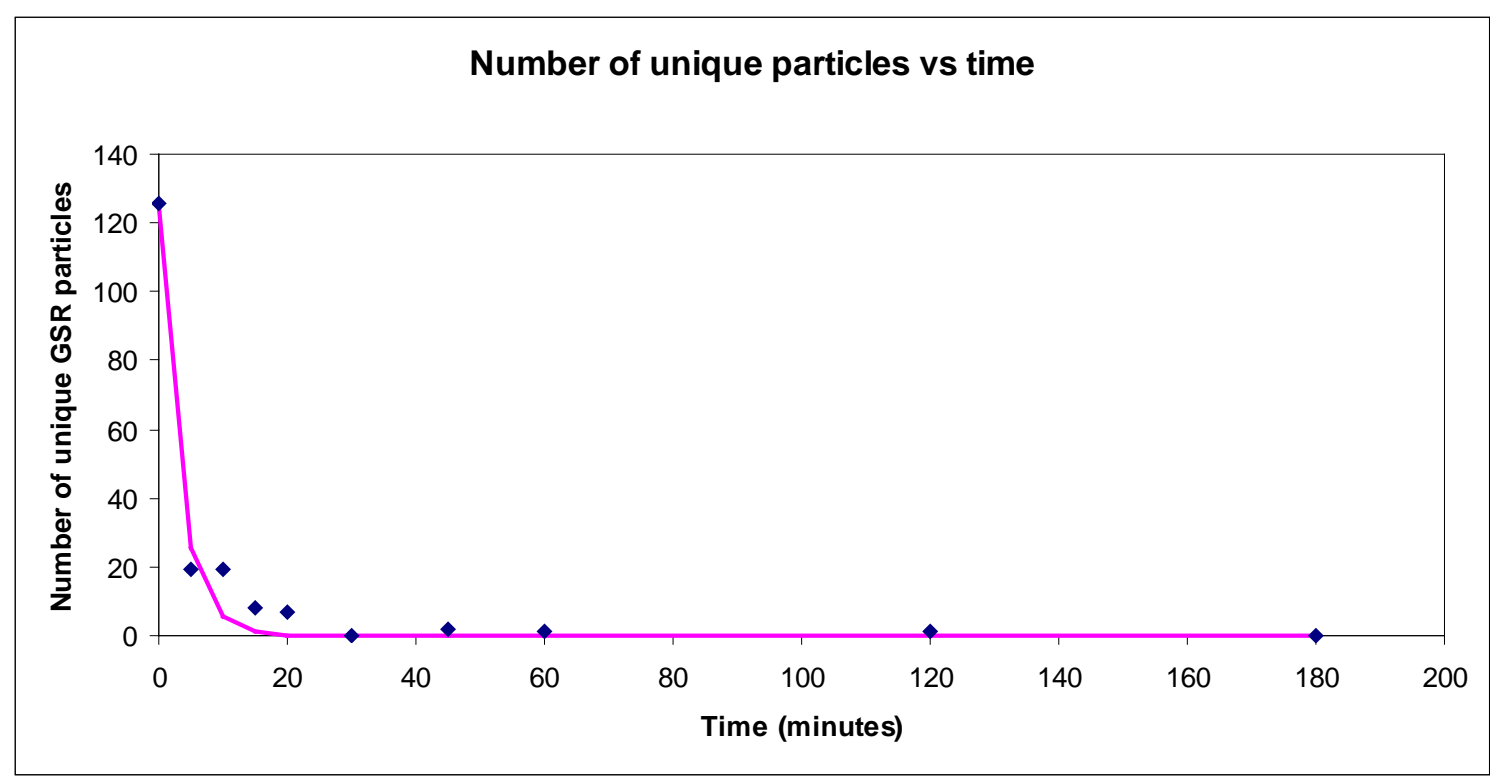

Figure 17: Number of unique GSR particles vs. time for participant 3 
For participant three $A_{0}$ was calculated to be 125.3 particles. Experimentally, 126 particles were found on the sample at time zero. The rate constant was determined to be 0.32. The goodness of fit for this analysis was determined to be 333.7. The largest contributor to this value was the datum at time zero. Given the large value for the goodness of fit, the fit line represented in Figure 17 does not represent that data very well. The reason for the large number of unique particles in this sample is unclear. When this datum was excluded from the analysis the value for $A_{0}$ was 30.8 and $\mathrm{k}$ was 0.076 . The goodness of fit improved from 333.7 to 41.2. These results are given in Figure 18.

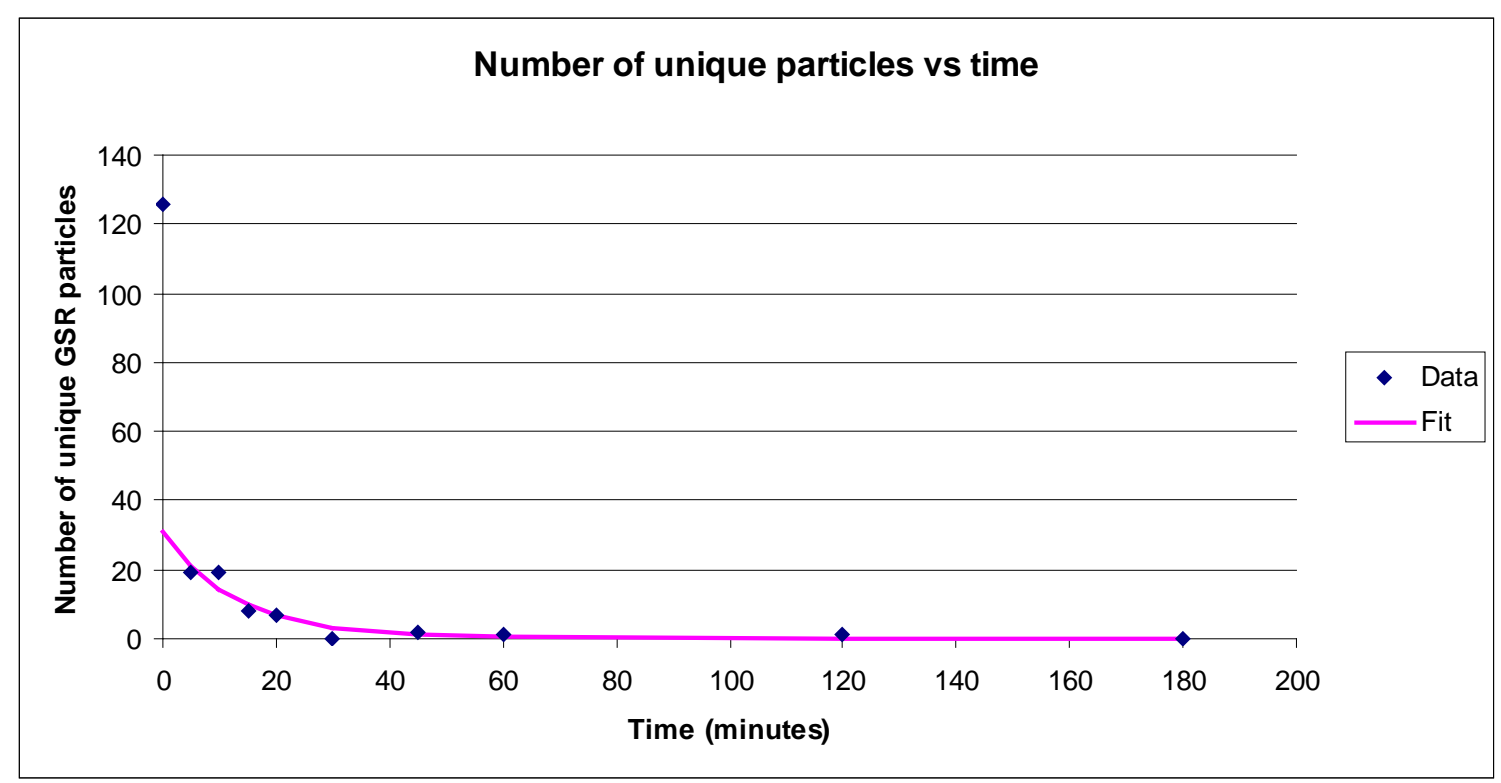

Figure 18: Number of unique GSR particles vs. time for participant 3 not including time zero datum point

Since both unique and indicative GSR particles result from the discharge of a firearm, one would expect that the first order kinetic model would be valid for the loss of indicative particles. It is unlikely that environmental and unclassified particles would 
follow the same decay trend based of the fact that the discharge of the firearm is not the sole source of these particles. Certain environmental particles such as spherical Pb (in the metallic state), are definitely products of a firearm discharge. This would imply that the total number of environmental particles on the hands of a shooter would increase after the discharge of the firearm and the number of these particles would decay over time to the background levels. For unclassified particles the background level will be dependent upon the normal environmental conditions of the individual. Since the deposition of environmental and unclassified particles on a person's hands is a dynamic process, such particles can be deposited from and lost to the environment from a person's hand. During the wait time of the volunteers unique and indicative particles are lost but environmentaland unclassified particles can be lost and gained.

Based on the above assumptions the first order kinetic decay model was used for the indicative particles represented in Table 14 and 15. There is a large variation in the number of indicative particles present at the various times. This fluctuation could be attributed to the relatively small number of indicative particles found in this experiment. Since each of these tests was performed sequentially, the level of activity during the wait time of this individual was similar. The situation was similar for participant three.

The inherent limitation of this type of study is in the assumption that each discharge of the firearm under similar conditions will result in the same number of GSR particles being deposited on the hands of the shooter. Each sample requires that a new shot be fired and the wait period increased to the prescribed time. If the number of particles deposited at the time of shooting remains constant then the comparison of a subsequent fire will provide the correct $A_{t} / A_{0}$ ratio. It is evident that for each shot the 
value of $A_{0}$ will vary. The implication is that $A_{t}$ is compared to $A_{0}$ of the first shot in the series. In general, the number of particles should remain constant and thus the approximated half-life should be a reliable value.

The following is for the indicative particles for participants 1 and 3 based one the first order kinetics decay model.

\begin{tabular}{|llll|}
\hline $\mathrm{k}$ & 0.03 & & \\
$\mathrm{~A} 0$ & 12.2 & half life & 26.8 \\
\hline
\end{tabular}

\begin{tabular}{|r|r|r|r|}
\hline \multicolumn{1}{|c|}{$\mathbf{t}$} & \multicolumn{1}{|c|}{ A } & \multicolumn{1}{c|}{ A calc } & \multicolumn{1}{c|}{ dos } \\
\hline 0.00 & 15.00 & 12.16 & 8.05 \\
\hline 5.00 & 7.00 & 10.69 & 13.62 \\
\hline 10.00 & 4.00 & 9.40 & 29.11 \\
\hline 15.00 & 10.00 & 8.26 & 3.04 \\
\hline 20.00 & 13.00 & 7.26 & 32.98 \\
\hline 30.00 & 8.00 & 5.61 & 5.73 \\
\hline 45.00 & 2.00 & 3.81 & 3.26 \\
\hline 60.00 & 0.00 & 2.58 & 6.67 \\
\hline 120.00 & 0.00 & 0.55 & 0.30 \\
\hline 180.00 & 0.00 & 0.12 & 0.01 \\
\hline & & & \\
\hline & & & 102.8 \\
\hline
\end{tabular}

Table 13: Calculated data from Excel for Participant 1 using first order kinetics for indicative GSR particles

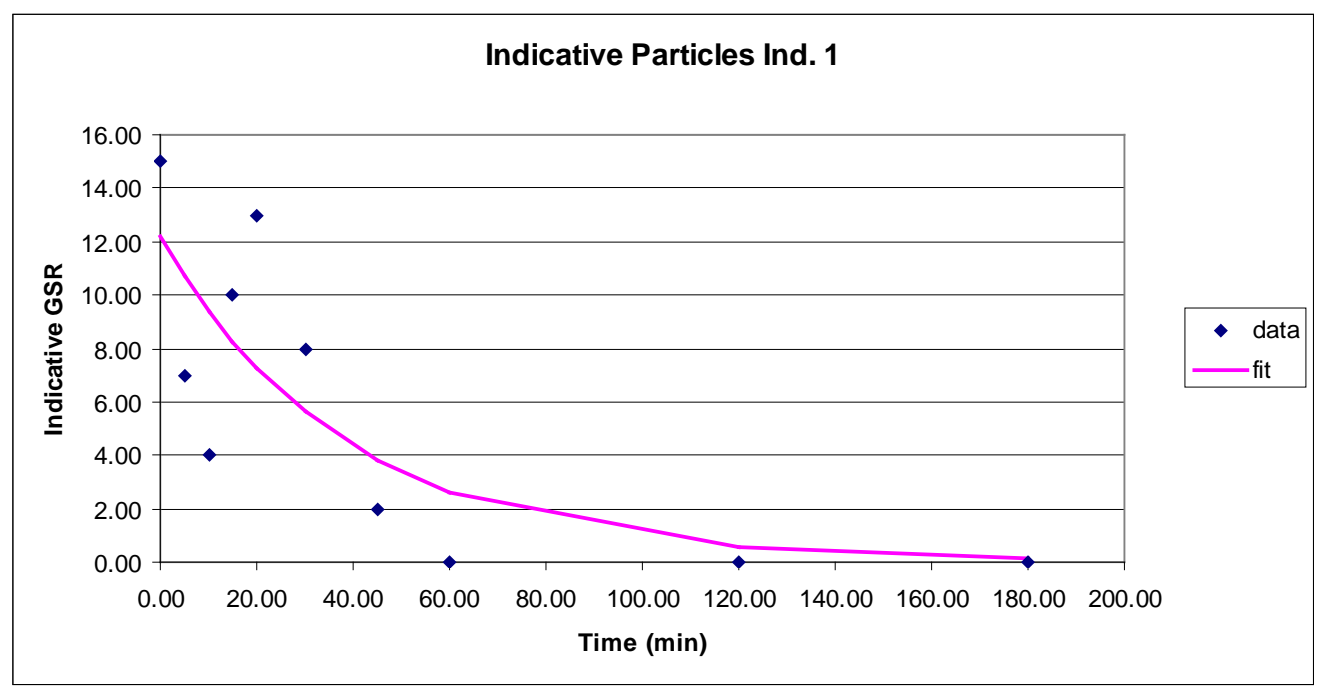

Figure 19: Number of indicative GSR particles vs. time for participant 1 


\begin{tabular}{|llll|}
\hline$k$ & 0.06 & & \\
$\mathrm{~A} 0$ & 25.3 & half life & 10.7 \\
\hline
\end{tabular}

\begin{tabular}{|r|r|r|r|}
\hline \multicolumn{1}{|c|}{$\mathbf{t}$} & \multicolumn{1}{|c|}{ A } & \multicolumn{1}{c|}{ A calc } & \multicolumn{1}{c|}{ dos } \\
\hline 0.00 & 30.00 & 25.25 & 22.53 \\
\hline 5.00 & 8.00 & 18.26 & 105.33 \\
\hline 10.00 & 16.00 & 13.21 & 7.80 \\
\hline 15.00 & 7.00 & 9.55 & 6.51 \\
\hline 20.00 & 17.00 & 6.91 & 101.86 \\
\hline 30.00 & 0.00 & 3.61 & 13.05 \\
\hline 45.00 & 0.00 & 1.37 & 1.87 \\
\hline 60.00 & 1.00 & 0.52 & 0.23 \\
\hline 120.00 & 0.00 & 0.01 & 0.00 \\
\hline 180.00 & 1.00 & 0.00 & 1.00 \\
\hline & & & \\
\hline & & & 260.18 \\
\hline
\end{tabular}

Table 14: Calculated data from Excel for Participant 3 using first order kinetics for indicative GSR particles

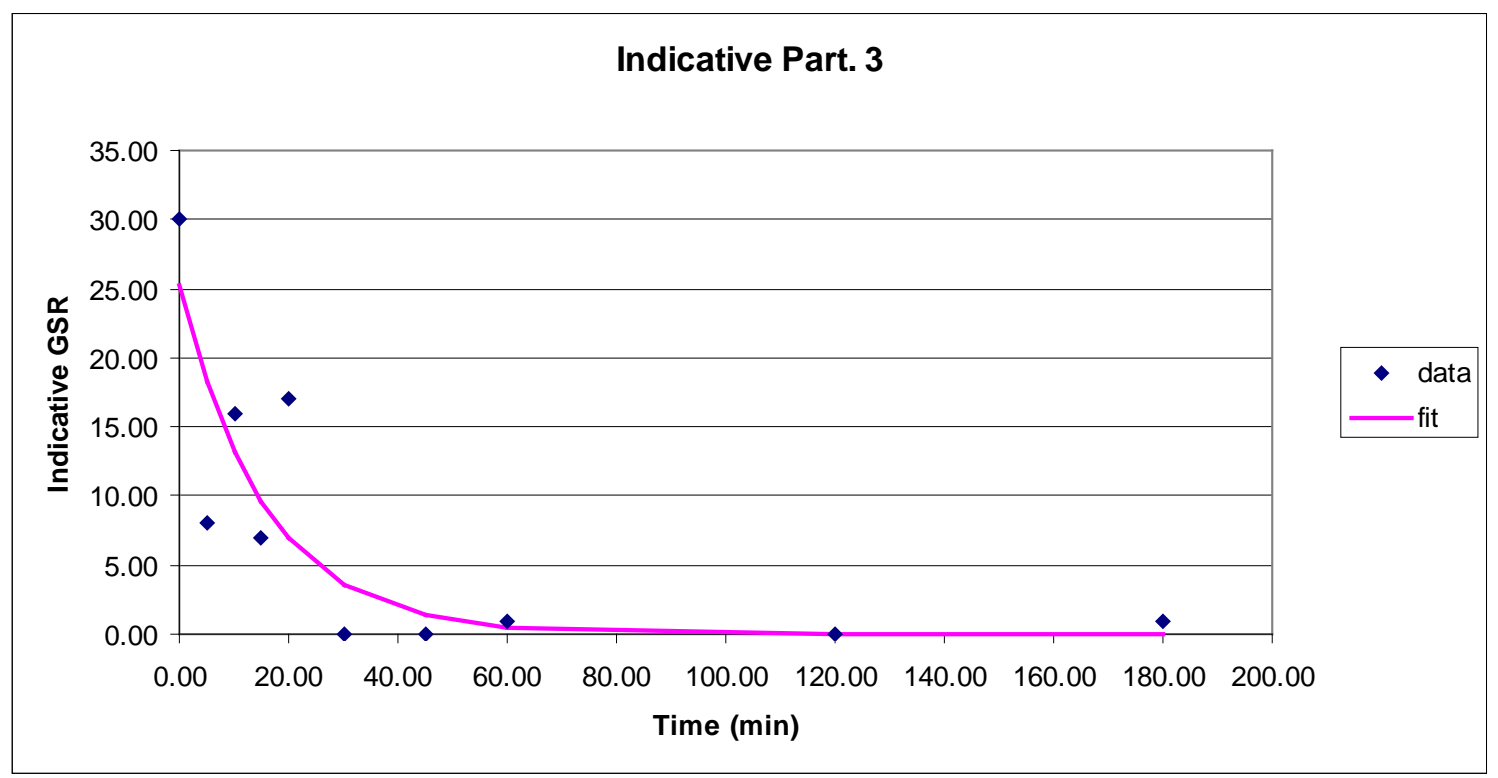

Figure 20: Number of indicative GSR particles vs. time for participant 3 
The average rate constant in this study is $0.053(.022)$. This equates to a half-life of 31 minutes. At a 95\% confidence level, the half-life would range between 7.1 minutes and 77 minutes. At 1 hour, 4\% of the original number of unique and indicative particles would remain. 


\subsection{Results from Automated Scanning}

The data acquired during the automated scans was analyzed using $\mathrm{R}$ and the functions rattle and lattice.

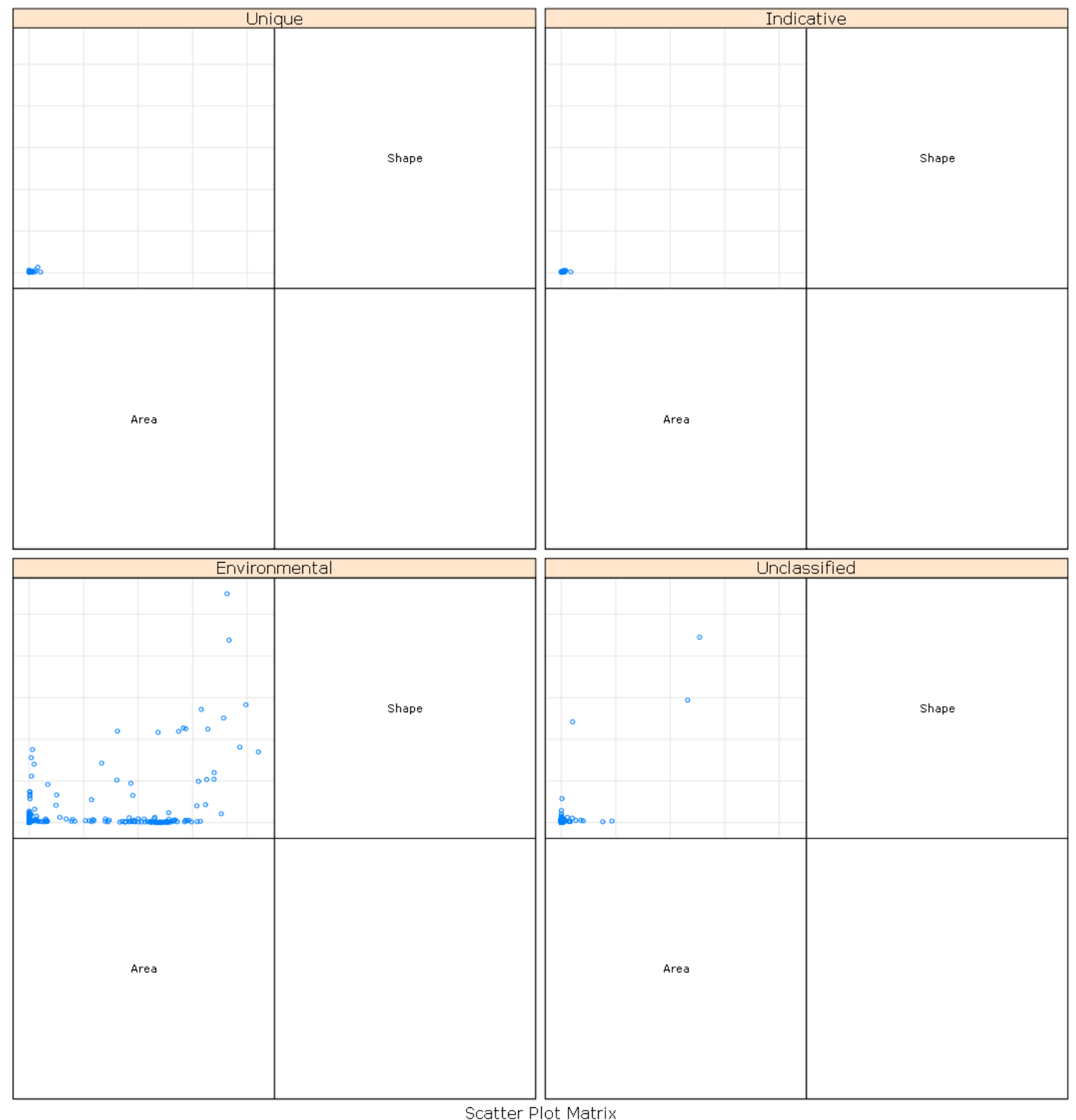

Figure 21: Scatter Plot of particle classifications looking at the relationship between area and shape of the particles 
The distribution of shape and area by particle class is given in Figure 21. The shape of a particle is defined as:

shape $=\frac{\text { perimeter }^{2}}{4 \pi \text { Area }}$

For a circle, the shape is 1.0. As the shape changes to an elongated rectangle this shape factor will increase. Unique and indicative particles it shows that all particles obtained for each classification were similar in shape and area based on the tight grouping in the corner of the box. This represents that unique and indicative particles have a specific size and shape. This supports our previous data and research that the shape and morphology of unique and indicative particles, that result from a discharge of a firearm is similar in shape, which is usually spherical.

Although looking at the environmental and unclassified particles if one looks at the shape and area there is a broad distribution of shape and area in the environmental particles. The majority along the bottom of the square have a similar shape but a wide distribution of size or area. Unclassified particles have a tighter grouping the in the bottom left corner showing they have a similar shape and size with a few outliers. 

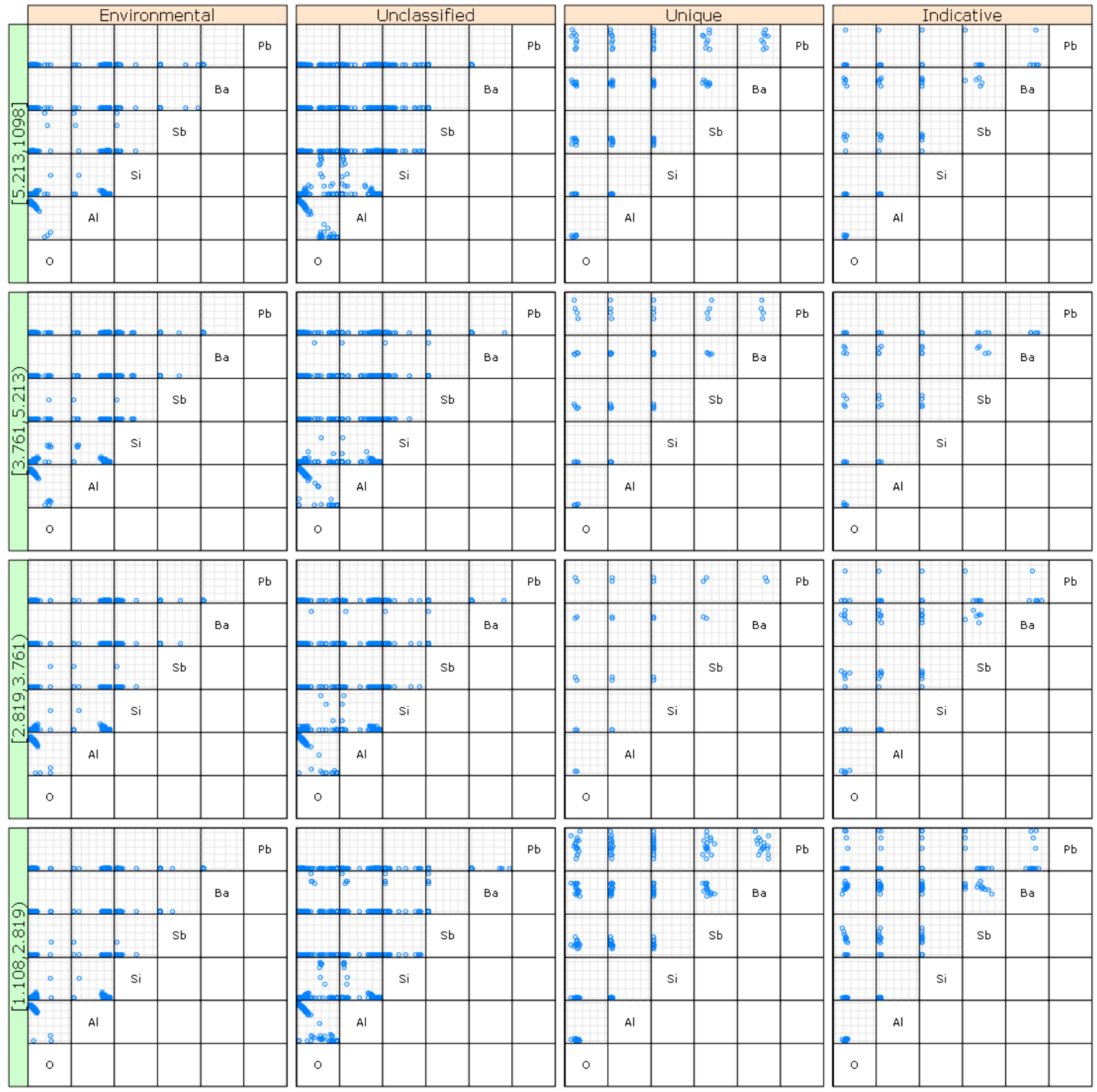

Figure 22: Scatter Plot of shape of the particle for each element in each particle classifications

A majority of GSR analysis is looking at morphology of particles so it was established that the automated data could compare the shape factor to the classification. 
Figure 22 shows the classification for particles along the top and down the side is the shape factor. Shape factor was established based on the equation perimeter ${ }^{2} / 4 \pi^{*}$ area.

A circle has a shape factor of 1 so the shape factor up the side shows how the shape of the particles relates to a circle or sphere. For example, if one looks at the unique particles in the highest shape factor $(5.213,10.98)$ one can see that for particles that contain $\mathrm{Pb}$ and $\mathrm{Ba}$ there is a large distribution of $\mathrm{Pb}$ and a tighter but still varied distribution of Ba. Using this data one can supports again the shape factor of unique and indicative particles having a similar shape. 

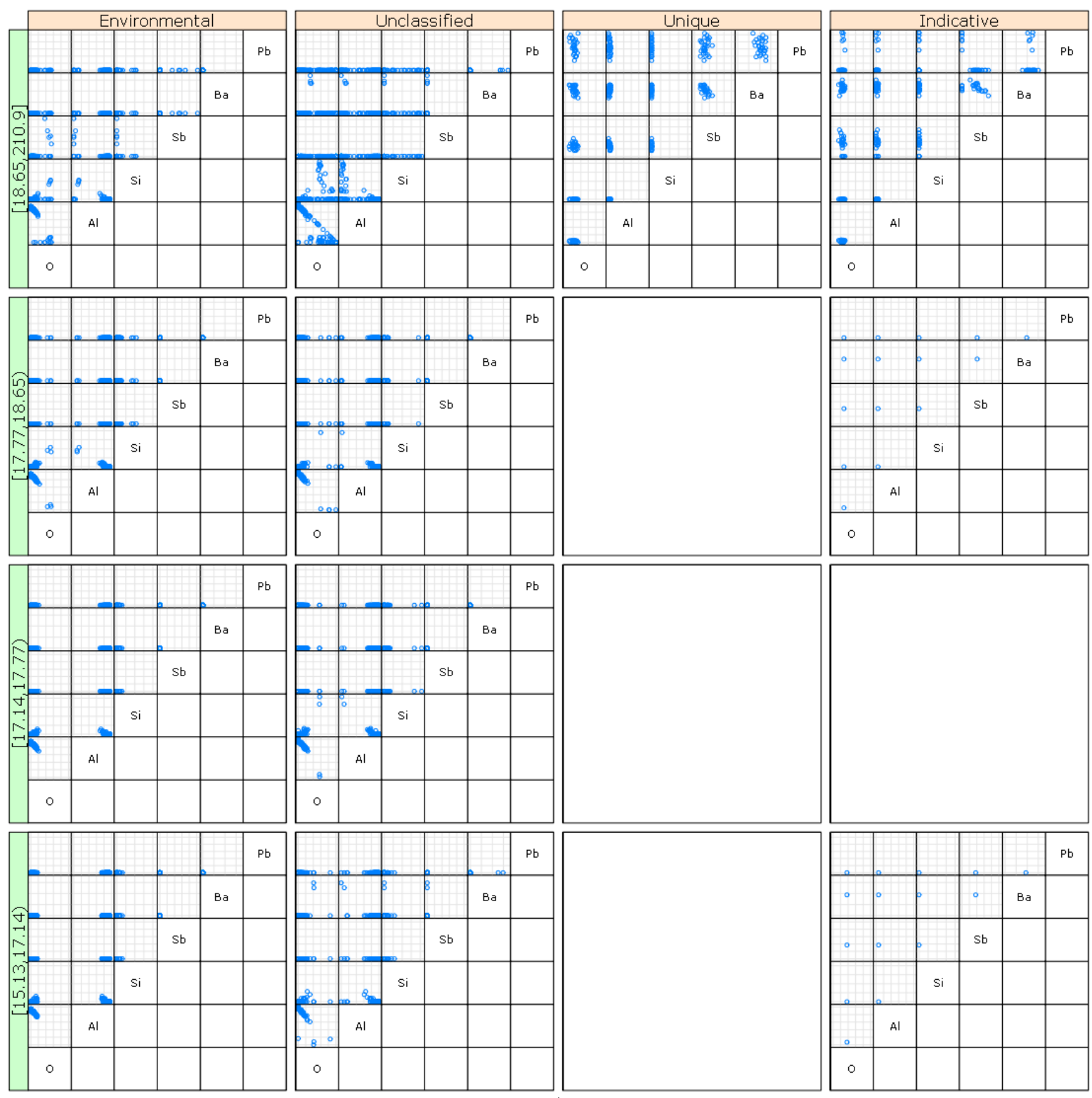

Figure 23: Scatter Plot of gray scale of the particle for each element in each particle classifications

Along with shape and size a large factor when running the analysis of GSR particles on the SEM/EDS is dealing with the grayscale. Figure 23 represents the grayscale ratios for each element contained in the four different classified particles. For example, looking at the unique GSR particles compared to the grayscale factor it can be 
established that unique particles are only seen in the highest grayscale range of (18.65, 210.9) this can be explained based on the elements contained in the unique particles. $\mathrm{Pb}, \mathrm{Ba}$, and $\mathrm{Sb}$ are all heavy elements that have higher atomic numbers which in return show up brighter in BSE detection so they fall in the top category for grayscale. There is no data for unique particles in the other three ranges of grayscales because the unique particles do not contain the lighter elements that show up in this grayscale range.

\subsection{Conclusion}

In conclusion, establishment of the fact that gunshot residue examination is an important factor in the forensic science field has been obtained. There is controversy over what is actually considered gunshot residue and what makes positive sample. However, it is well used and is beneficial to forensic science. After the study of persistence was completed, it was found that based on first order kinetics there is a predicted amount of decay over time based on the number of initial particles found. The results of the research indicate that after approximately twenty minutes there was a great loss in the number of positive particles found on the hands. After approximately fortyfive minutes there were no positive GSR particles left on the hands of the shooter. This information can be used by law enforcement agencies to establish a working idea of how long it is necessary for samples to be taken after an incident. For example, is it necessary to take gunshot residue samples from a suspect in a crime that occurred two or three hours ago? This information could help determine a time frame for necessary sampling. 
Further work with this study is needed. More time would have allowed more extensive analysis of the samples that were analyzed along with time to analyze the other samples collected. In the future, analysis of samples taken from studies two and three need to be analyzed and interpreted. This data may help scientists establish a certain number of particles needed to classify a sample as being positive for GSR. There is great potential for future work with this study. 


\section{References}

1. Schwoeble AJ, Exline DL. Current Methods of Forensic Gunshot Residue Analysis: Chapter 1. Current Methods in Forensic Gunshot Residue Analysis: CRC Press LLC; 2000;9.

2. Meng H-h, Caddy B. Gunshot Residue Analysis A Review. Journal of Forensic Science. 1997;42(4):553-70.

3. Wolten GM, Nesbitt RS, Calloway AR, Loper GL, Jones PF. Particle Analysis for the Detection of Gunshot Residue. I: Scanning Electron Microscopy/Energy Dispersion X-Ray Characterization of Hand Deposits from Firing. Journal of Forensic Science. 1979 April 1979;24(2):409-22.

4. Berk RE, Rochowicz SA, Wong M, Kopina MA. Gunshot Residue in Chicago Police Vehicles and Facilities: An Empirical Study. Journal of Forensic Science. 2007 July 2007;52(4):838-41.

5. Fojtásek L, Vacínová J, Kolár P, Kotrlý M. Distribution of GSR particles in the surroundings of shooting pistol. Forensic Science International. 2003;132(2):99-105.

6. MacCrehan WA, Layman MJ, Secl JD. Hair combing to collect organic gunshot residues (OGSR). Forensic Science International. 2003;135(2):167-73.

7. Fojtásek L, Kmjec T. Time periods of GSR particles deposition after dischargefinal results. Forensic Science International. 2005;153(2-3):132-5.

8. Schwoeble AJ, Exline DL. Current Methods in Forensic Gunshot Residue Analysis: Chapter 3. Current Methods in Forensic Gunshot Residue Analysis: CRC Press LLC; 2000;15.

9. Goldstein J, Newbury D, Joy D, Lyman C, Echlin P, Lifshin E, et al. Scanning Electron Miscroscopy and X-ray Microanalysis. Third ed. Ney York, NY: Springer Science + business Media, Inc., 2003.

10. Bertin E. Introduction to X-ray Spectrometric Analysis. New York: Plenum Press, 1979.

11. The Electron Probe Technique [database on the Internet]. 2006 [cited 04/19/09]. Available from: http://epmalab.uoregon.edu/epmatext.htm.

12. SEM/EDS: Scanning Electron Microscopy with X-ray microanalysis [database on the Internet]. [cited 04/19/09]. Available from: http://www.sdm.buffalo.edu/scic/semeds.html. 
13. Ltd OIA. INCAPentaFET-x3. 2006 [updated 2006; cited 04/28/2010]; Available from.

14. Cowan ME, Purdon PL. A Study of the "Paraffin" Test. Journal of Forensic Sciences. 1967;12(1):18.

15. Wallace JS. Chemical Analysis of Firearms, Ammunition and Gunshot Residue: Chap 17 Taylor and Francis Group, LLC

16. Wolten GM, Nesbitt RS, Calloway AR. Particle Analysis for the Detection of Gunshot Residue. III: The Case Record. Journal of Forensic Science. 1979 October 1979;24(4):864-9.

17. Zumdahl SS. Chemistry. Fourth ed. Boston New York: Houghton Miffline Company, 1997.

18. Krishnan SS. Detection of Gunshot Residues on the Hands by Trace Element Analysis. Journal of Forensic Science. 1977;22(2):304-24.

19. Wessel J, Jones P, Kwan Q, Nesbitt R, Rattin E. Equipment systems improvement program gunshot residue detection. The Aerospace Corporation. 1974.

20. Basu S. Formation of Gunshot Residues. Journal of Forensic Science. 1982;27(1):72-91.

21. Association IHE. 2002 [updated 2002; cited 04-10-10]; Available from: http://homestudy.ihea.com/aboutfirearms/05a_howfires.htm.

22. Newton JT. Rapid Determination of Antimony, Barium, and Lead in Gunshot Residue Via Automated Atomic Absorption Spectrophotometry. Journal of Forensic Science. 1981;26(2):302-12.

23. Romolo FS, Margot P. Identification of gunshot residue: a critical review. Forensic Science International. 2001;119(2):195-211.

24. Pillay KKS, Jester WA, Fox HA. New Method for the Collection and Analysis of Gunshot Residues as Forensic Evidence. Journal of Forensic Science. 1974;19(4):768-83.

25. Steinburg M, Leist Y, Tassa M. A New Field Kit for Bullet Hole Identification. Journal of Forensic Sciences. 1984;29(1):169-76.

26. Harrison HC, Gilroy R. Firearms Discharge Residues. Journal of Forensic Science. 1959;4(2):184.

27. Feigl F. Spot tests in organic analysis. VII ed. Amsterdam: Elsevier, 1966. 
28. Maloney RS, Thornton JI. Colour test with diphenylamine stabiliser and related compounds in smokeless gunpowder. Journal of Forensic Science. 1982;27:318-29.

29. Krishnan SS. Detection of Gunshot Residue on the Hands by Neutron Activation and Atomic Absorption Analysis .Journal of Forensic Science. 1974;19(4).

30. Ruch RR, Buchanan JD, Guinn SC, Bellanca RH, Pinker. Application of neutron activation analysis in scientific crime detection. Journal of Forensic Science. 1964;9:11932.

31. Krishnan SS. Rapid Detection of Firearm Discharge Residues by Atomic Absorption and Neutron Activation Analysis. Journal of Forensic Science. 1971;16.

32. Stone I, Petty C. Examination of Gunshot Residues. Journal of Forensic Sciences. 1974;19(4):784-8.

33. Jones PF, Nesbitt RS. A photoluminescence technique for the detection of gunshot residues. Journal of Forensic Science. 1975;20:231-42.

34. Nesbitt RS, Wessel JE, Wolten G, Jones PF. Evaluation of a Photoluminescence Technique for the Detection of Gunshot Residue. Journal of Forensic Science. 1977;22(2):288-303.

35. Kilty JW. Activity After Shooting and Its Effect on the Retention of Primer Residue. Journal of Forensic Science. 1975;20(2):219-30.

36. MacCrehan WA, Smith KD, Rowe WF. Sampling Protocols for the Detection of Smokeless Powder Residues Using Capillary Electrophoresis. Journal of Forensic Science. 1998 43(1):119-24.

37. Wolten GM, Nesbitt RS, Calloway AR, Loper GL, Jones PF. Particle Analysis for the Detection of Gunshot Residue. II: Occupational and Environmental Particles. Journal of Forensic Science. 1979 April 1979;24(2):423-30.

38. VIM DM. Gunshot wounds: CRC Press, 1993.

39. DiMaio VJM, Dana SE, Taylor WE, Ondrusek J. Use of Scanning Electron Microscopy and Energy Dispersive X-Ray Analysis (SEM-EDXA) in Identification of Foreign Material on Bullets. Journal of Forensic Science. 1987;32(1):38-47.

40. Basu S, Boone CE, Denio DJ, Miazga RA. Fundamental Studies of Gunshot Residue Deposition by Glue-Lift. Journal of Forensic Science. 1997;42(4):571-81.

41. Lebiedzik J, Johnson DL. Rapid Search and Quantitative Analysis of Gunshot Residue Particles in the SEM. Journal of Forensic Sciences. 2000 January 2000;45(1):8392. 
42. White RS, Owens AD. Automation of Gunshot Residue Detection and Analysis by Scanning Electron Microscopy/Energy Dispersive X-Ray Analysis (SEM/EDX). Journal of Forensic Sciences`. 1987;32(6):1595-603.

43. Matricardi VR, Kilty JW. Detection of Gunshot Residue Particles from the Hands of a Shooter. Journal of Forensic Science. 1977;22(4):725-38.

44. Tillman WL. Automated Gunshot Residue Particle Search and Characterization. Journal of Forensic Sciences. 1987;32(1):62-71.

45. Andrasko J, A.Maehly. Detection of Gunshot Residues on Hands by Scanning Electron Microscopy. Journal of Forensic Science. 1977;22(2):279-87.

46. Gialamas DM, Rhodes EF, D.Crim, Sugarman LA. Officers, Their Weapons and Their Hands: An Empirical Study of GSR on the Hands of Non-Shooting Police Officers. Journal of Forensic Science. [Technical Note]. 1995 November 1995;40(6):1086-9.

47. Jalanti T, Henchoz P, Gallusser A, Bonfanti MS. The persistence of gunshot residue on shooters' hands. Science \& Justice. 1999;39(1):48-52.

48. Nesbitt RS, Wessel JE, Jones PF. Detection of Gunshot Residue by Use of the Scanning Electron Microscope. Journal of Forensic Science. 1976;21(3):595-610.

49. Zeichner A, Levin N. Collection Efficiency of Gunshot Residue (GSR) Particles from Hair and Hands Using Double-Side Adhesive Tape. Journal of Forensic Science. 1993;38(3):571-84.

50. Zeichner A, Levin N. Casework Experience of GSR Detection in Israel, on Samples from Hands, Hair, and Clothing Using an Autosearch SEM/EDX System. Journal of Forensic Science. 1995;40(6):1082-5.

51. Heard B. Handbook of Firearms and Forensic ballistics. Chichester: John Wiley \& sons, 1997.

52. Murdock J. The collection of gunshot discharge GSRs. Association of Firearms and Tool Mark Examiners Journal. 1984;16(3):136-41. 


\section{Appendix A}

\begin{tabular}{|c|c|c|c|c|c|c|c|c|c|c|c|c|c|}
\cline { 2 - 17 } \multicolumn{1}{c|}{} & \multicolumn{3}{c|}{ Unique } & \multicolumn{4}{|c|}{ Indicative } & \multicolumn{5}{c|}{ Environmental } \\
\hline Stub & SbBaPb & TiZnCu & SbBa & SbPb & BaPb & TiZn & Sb & Ni & $\mathrm{Pb}$ & Sn & Au & Fe & $\mathrm{Cu}$ \\
\hline 5 & 1 & 0 & 0 & 0 & 0 & 0 & 0 & 1 & 2 & 0 & 0 & 5 & 2 \\
\hline 6 & 20 & 0 & 12 & 3 & 0 & 0 & 2 & 1 & 2 & 0 & 0 & 28 & 21 \\
\hline 7 & 24 & 0 & 8 & 4 & 0 & 1 & 0 & 3 & 5 & 0 & 1 & 37 & 35 \\
\hline 8 & 12 & 0 & 4 & 0 & 0 & 0 & 0 & 0 & 6 & 0 & 0 & 9 & 32 \\
\hline 9 & 35 & 1 & 20 & 9 & 5 & 0 & 5 & 3 & 22 & 0 & 0 & 114 & 65 \\
\hline 10 & 10 & 0 & 8 & 4 & 1 & 0 & 2 & 4 & 2 & 1 & 0 & 42 & 29 \\
\hline 11 & 5 & 0 & 5 & 3 & 0 & 0 & 1 & 2 & 4 & 0 & 0 & 28 & 21 \\
\hline 12 & 1 & 0 & 2 & 0 & 0 & 0 & 0 & 0 & 0 & 0 & 0 & 27 & 10 \\
\hline 13 & 0 & 0 & 0 & 0 & 0 & 0 & 0 & 0 & 0 & 0 & 0 & 5 & 0 \\
\hline 14 & 0 & 0 & 0 & 0 & 0 & 0 & 0 & 6 & 0 & 0 & 0 & 4 & 0 \\
\hline 15 & 0 & 0 & 0 & 0 & 0 & 0 & 0 & 0 & 0 & 0 & 0 & 2 & 2 \\
\hline
\end{tabular}

Appendix A: Manual analysis data from Participant 1 


\section{Appendix B}

\begin{tabular}{|c|c|c|c|c|c|c|c|c|c|c|c|c|c|c|}
\hline & Unique & \multicolumn{9}{|c|}{ Indicative } & \multicolumn{6}{|c|}{ Environmental } \\
\hline Stub & SbBaPb & SbBa & $\mathrm{SbPb}$ & $\mathrm{BaPb}$ & $\mathrm{SbSn}$ & $\mathrm{Ti} \mathrm{Zn}$ & $\mathrm{Sb}$ & $\mathrm{Cu} \mathrm{Zn}$ & $\mathrm{Ni}$ & $\mathrm{Pb}$ & $\mathrm{Sn}$ & $\mathrm{Au}$ & $\mathrm{Fe}$ & $\mathrm{Cu}$ \\
\hline 49 & 2 & 0 & 1 & 0 & 0 & 0 & 0 & 5 & 0 & 3 & 0 & 0 & 5 & 7 \\
\hline 50 & 125 & 21 & 4 & 3 & 2 & 0 & 0 & 36 & 9 & 1 & 0 & 0 & 17 & 104 \\
\hline 51 & 19 & 7 & 0 & 1 & 0 & 0 & 0 & 25 & 1 & 1 & 0 & 0 & 16 & 16 \\
\hline 52 & 19 & 12 & 4 & 0 & 0 & 0 & 1 & 14 & 6 & 9 & 0 & 0 & 20 & 31 \\
\hline 53 & 8 & 2 & 2 & 1 & 0 & 2 & 1 & 10 & 3 & 2 & 2 & 0 & 15 & 14 \\
\hline 54 & 7 & 12 & 1 & 0 & 0 & 4 & 1 & 7 & 0 & 1 & 0 & 2 & 21 & 26 \\
\hline 55 & 0 & 0 & 0 & 0 & 0 & 0 & 0 & 1 & 0 & 0 & 0 & 0 & 0 & 0 \\
\hline 56 & 2 & 0 & 0 & 0 & 0 & 0 & 0 & 2 & 0 & 2 & 0 & 0 & 4 & 4 \\
\hline 57 & 1 & 1 & 0 & 0 & 0 & 0 & 0 & 1 & 0 & 0 & 0 & 0 & 8 & 3 \\
\hline 58 & 1 & 0 & 0 & 0 & 0 & 0 & 0 & 3 & 1 & 0 & 0 & 0 & 3 & 2 \\
\hline 59 & 0 & 1 & 0 & 0 & 0 & 0 & 0 & 1 & 1 & 0 & 0 & 0 & 1 & 3 \\
\hline
\end{tabular}

Appendix B: Manual analysis data from Participant 3 